\title{
Órbitas Periódicas en el Problema de Störmer
}

Lidia Jiménez Lara

29 de Abril de 1991 


\section{Capítulo 1}

\section{Introducción}

El problema de determinar el movimiento de una partícula cargada en el campo de un dipolo magnético puntual es muy antiguo y ha sido de mucho interés debido a que es la primcra aproximación para el estudio del movimiento de partículas cargadas en el campo magnético de la ticra. El problema fué formulado por primera vez por Störmer [39] en 1907, y posteriormente cstudiado por él y otros deljido a su conexión con las auroras polares, la radiación cósmica y los cinturones de Van Allen. Ilay gran cantidad de literatura acerca de este problema. En la bibliugrafía enlisto algunas referencias importantes.

El movimiento de una partícula en un campo magnético es por lo general muy complicado y debe obtenerse por integración numérica de las ecuaciones diferenciales de movimiento. Dragt y Finn [12] mostraron desde diferentes puntos de vista por qué puede esperarse que el problema de Störmer no tenga más int.cgrales de movimicnto que la energía y la proyección del momento angular en la dirección del dipolo. Una demostración rigurosa sobre la no integrabilidlad fué dada por Noguera [34] en un caso particular. É] demuestra la incusión del shift de Bernoulli como un subsistema de las variedades invariantes de la órbita de Lyapunov en el punto de equilibrio aislado (ver capítulo 3).

En el caso especial de un campo magnético uniforme, las trayectọias se pueden obtener explícitamente. Como se sabe, las partículas giran en una. hélice alrededor de las líneas de campo magnélico, con frecuencia y radio de giro constantes. De mucho interés ha sido el movimiento de partículas cargadas en campos que varían lentamente (comparado con el radio de giro y el período). Esto significa que en el transcurso de un giro alrededor de 


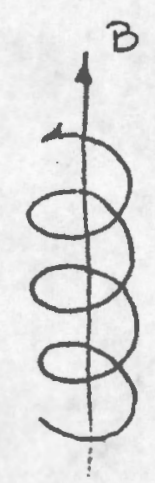

Figura 1.1: Carga eléctrica cn un campo magnélico constante

una línea de campo, la partícula ve un campo aproximadamente constante, moviéndose aproximadamente en un círculo cuyo centro se desplaza lentamente a lo largo de las líneas de fuerza y se mueve rájidanente alrededor de las líneas. A ésta aproximación se le llama adiabática o del centro guía [1], [19], [35]. En campos magnéticos donde las líneas de campo convergen (espejos magnét.jcos), las partículas cargadas se reflcjan en las regiones de campo magnélico fuerte $y$ puede esperarse un moviniento atrapado entre dos puntos espejo.

Braun [5] demostró que el cspacio fase de una partícula movićndose bajo la influencia de un campo magnético de un dipolo contiene una región que incluye la región adiabática, donde las partículas están atrapadas para todo tiempo. La base de esta demostración es usar un teorema de Moser que garantiza la existencia de soluciones cuasi-periódicas de las ecuaciones diferenciales de movimiento. Así, muestra que las parlículas que están atrapadas adiabáticannente están de liecho atrapadas para todo tiempo.

Vallarta y lemaitre [24], [12] estudiaron el prob]ema de Störmer y calcularon la oricntación en la cual podían incidir las partículas cósmicas sobre la superficie terrestre, llamado el cono permitido de la radiación cósmica.

Graef y Kusaka [16] resolvieron el caso ecuatorial explícitamcnte en términos de funciones elípticas. Sin enbargo, el problema tridimensional no es integrable. Frecuentemente se restringe el estudio del problema de Störmer al movimiento en cl plano mcridiano usando el hecho de que la variable ažimutal es ignorable en el Ilamiltoniano. El problema queda reduciclo a un sistenia con dos grados de libertad y una constante de movimiento (ver capítulo 2). Markellos y sus colaboradores estudiaron este caso [15], [27]- [33] y obtuvieron órbitas periódicas simétricas simples y múltiples en el plano meridiano, así 
como sus parámetros de estabilidad e hicieron una clasificación cn familias. Estudiaron tambićn la familia asimćtrica principal [29] (ver capítulo 5).

Anteriormente, De Vogelaere [11] (basado en las ideas de Birkholl [2]) habia introducido el concepto de lineas de simetría en mapeos de l'oincaré para obtener órbitas periódicas. Aunque este método fué poco usado en el pasado, ha resurgido recientenent,e como un poderoso método para obtener órbitas periódicas y comprender mejor la dinánica de los sistemas dinámicos con dos grados de libertad y una integral de movimiento: el mapeo estándard [23] [36], el problema de tres cuerpos [7], el estudio de caos en dispersión clásica para cl problema de Stömmer [22], el trompo simétrico con un punto fijo en un campo gravitacional constante [S], el estudio del mapeo cuasicristal [37], el estudio de una bola de billar moviéndose entre una pared vertical y un plano inclinalo bajo la acción del campo gravilacional [38], y finalmente en el estudio y clasificación de órbitas periódicas en el probjema de Störmer $[20],[21]$, que se presenta cn esta tesis.

En este tral bajo utilizo el mótodo de De Vogelace para estudiar el movimiento en cl plano meridiano, calculando varias líneas de simetría en el plano de Poincaré (ecuatorial) para diferentes valores del parámetro de Störmer. Queda aqui manifiesta la importancia de las lineas de simetría pucs sincetiza muchos trabajos anteriores sobre bifurcaciones y aparición de órbitas periódicas. Además proporciona una clasificación natural en familias.

En el capítulo 2 presento las ecuaciones de movimiento del problema. Se analiza el caso ccuatorial y se plantean las ecuaciones de movimiento en el plano meridiano. En el capítulo 3 presento una descripción cualitativa del movimiento y calculo las curvas de velocidad cero en el plano meridiano. En el capítulo 4 utilizo el plano ecuatorial para introducir el mapeo de Poincaré y una factorización de este mapeo en el producto de dos involuciones básicas. Se definen entonces un conjunto infinito de involuciones en térninos de las básicas, asi como su conjunto de puntos fijos (líneas de simetría) y se demuestran varias propiedades importantes. En el capilulo 5 presento un análisis de la dinámica de ciertas líneas de simetría para estudiar la llamada familia principal y entender la clasificación de las órbitas periódicas simples (períodos 1 y 2) en el problema de Störmer, (incluyendo las asimétricas simples). Se hace una correspondencia con los trabajos de Markellos y sus colaboradores. Finalmente, cn el capítulo 6 estudio las órbilas de periodo 3, para las que obtengo la estructura global en el espacio de parámetros. 


\section{Capítulo 2}

\section{Ecuaciones de movimiento}

Considérese una carga $q$ de masa m moviéndose en el campo de un dipolo magnético de momento $\mathbf{M}$, conı se muestra en la figura (2.1).

El Hamiloniano es:

$$
I_{1}=\frac{1}{2 m}[\mathbf{P}-q \mathbf{A}]^{2}
$$

con

$$
m \dot{\mathbf{r}}=\mathbf{P}-q \mathbf{A}
$$

donde $\mathbf{r}$ es el vector de posición de la carga medido desde el dipolo (supuesto puntual); $\mathbf{P}$ es su momento conjugado y $\mathrm{A}=(\mathbf{M} \times \mathbf{r}) / r^{3}$, el potencial dipolar.

Nótese que $v=|\dot{\mathbf{r}}|=\sqrt{2 H_{1} / m}$ es constante. El que la energía cinética. se conserve se debe al hecho de que la fuerza de Lorentz es perpendicular al movimiento de la partícula y entonces no efectúa trabajo. Se tienen entonces dos constantes de movimiento: el Hamiltoniano (debido a que es indepen-

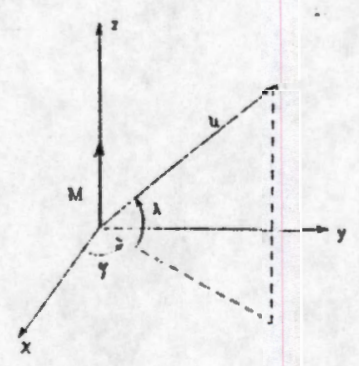

Figura 2.1: Coordenadas de la carga en el campo magnético dipolar 
diente del ticmpo), y la proyección del momento angular en la dirección de M.

Introduzco ahora un cambio a variables adimensionales, originalnente debido a Störmer, que climina todas las cantidades físicas que entran cn estas ecuaciones ( $\mathrm{m}, \mathrm{M} \mathrm{y}$ q). Esto se logra utilizando como unidad de longitud la. cantidad

$$
C_{1}=\sqrt{\frac{M|q|}{m v}}
$$

e introduciendo las variables adimensionales u y s, definidas por:

$$
\mathbf{r}=\mathbf{u} C_{1} \quad v t=s C_{1}
$$

En estas variables el Ilamiltoniano resulta:

$$
H=\frac{1}{2}\left[\mathbf{p}-\frac{\hat{\mathbf{M}} \times \mathbf{u}}{u^{3}}\right]^{2}
$$

con

$$
\mathbf{u}^{\prime}=\mathbf{p}-\hat{\mathbf{M}} \times \mathbf{u} / \mathbf{u}^{3}
$$

donde $\mathbf{p}$ es cl momento conjugado a $\mathbf{u}, \mathrm{y} \mathbf{u}^{\prime}=d \mathbf{u} / d s$ es unitario ya que:

$$
\frac{d \mathrm{r}}{d t}=v \frac{d \mathrm{u}}{d s}
$$

así, $H$ de la ecuación (2.2) toma numéricamente el valor $1 / 2$.

En seguida introduzco coordenadas esféricas $(u, \lambda, \varphi)$ con el eje $z$ en la dirección del momento magnético dipolar, siendo $\lambda$ la latitud o variable zenital y $\varphi$ la longitud o variable azimutal (figura (2.1)). El Hamiltonjano es:

$$
H=\frac{1}{2}\left[p_{u}{ }^{2}+\frac{p_{\lambda}^{2}}{u^{2}}+\frac{\left(p_{\varphi}-\cos ^{2}(\lambda) / u\right)^{2}}{u^{2} \cos ^{2}(\lambda)}\right]=\frac{1}{2}
$$

donde puede observarse directamente la conservación del momento angular en la dirección del dipolo, ya que $H$ no depende explícitamente de $\varphi$. De las ecuaciones de Hamilton se obtiene que:

$$
p_{\varphi}=u^{2} \cos ^{2}(\lambda) \frac{d \varphi}{d s}+\frac{\cos ^{2}(\lambda)}{u}=2 \gamma_{1}
$$

donde se la definido a $\gamma_{1}$ en términos de la constante $p_{\varphi}$. $\gamma_{1}$ se conocc como el parámetro de Störmer y será de mucha importancia en este trabajo. 
Una consecuencia inmediata de la cxistencia de la integral (2.4) es que el movimiento de una particula en el espacio pucde resolverse como un movimiento con dos componentes:

1. Un moviniento en el plano meridiano con coordenadas $(u, \lambda)$ gobernado por las ecuaciones

$$
\begin{array}{r}
u^{\prime \prime}-u \lambda^{\prime 2}-\frac{\left(2 \gamma_{1}\right)^{2}}{u^{3} \cos ^{2} \lambda}+\frac{6 \gamma_{1}}{u^{4}}-\frac{2 \cos ^{2} \lambda}{u^{5}}=0 \\
u \lambda^{\prime \prime}+2 u^{\prime} \lambda^{\prime}-\frac{\sin \lambda \cos \lambda}{u^{5}}+\frac{4 \gamma_{1}^{2} \sin \lambda}{u^{3} \cos ^{2} \lambda}=0
\end{array}
$$

donde prima significa derivada respecto de $s$.

2. El movinicuto de rotación del plano meridiano alrededor del eje $z$ (dirección de M). Essta rotación estará dada por

$$
\varphi^{\prime}=\frac{2 \gamma_{1}}{u^{2} \cos ^{2} \lambda}-\frac{1}{u^{3}}
$$

Supóngase que se lian resuelto las ecuaciones (2.5) y (2.6), de manera que el movimiento cn el plano meridiano es conocido. Para especificar completamente la dirección de la partícula viajando en el espacio, se necesitarán conocer dos angulos [42], que pueden ser el ángulo 0 entre la tangente de la. trayectoria $\tau$ en el espacio y el plano meridiano $\mathrm{M}$, y el ángulo $\eta$ que forma. la proyección de $\tau$ sobre $M$ con la dirección del zenit (dirección de $i_{p}$ en la figura (2.2)).

Puede demostrarse [42] que:

$$
\pm \sin \theta=\frac{2 \gamma_{t}}{u \cos \lambda}-\frac{\cos \lambda}{u^{2}}
$$

donde + es para partículas positivas y - para negativas, y que

$$
\text { l,a.n } \eta=u \frac{d \lambda}{d u}
$$

Por lo tanto, si se conoce la trayectoria en el plano meridiano, su dirección en el espacio en cualquier punto se podrá conocer mediante (2.8) y (2.9) sin necesidad de resolver la ecuación (2.7). 


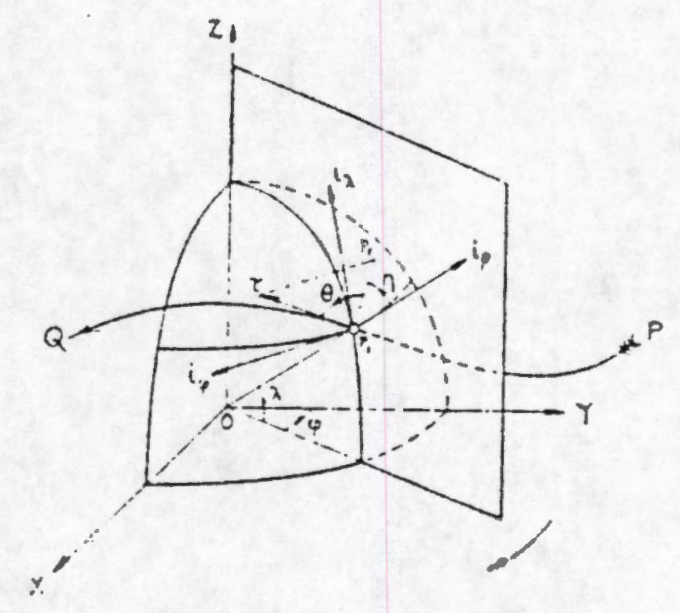

Figura 2.2: Angulos 0 y $\eta$.

\subsection{Movimiento en el plano ecuatorial}

Si el movimiento se limita al plano ecuatorial $\lambda=0$, habrán dos constantes de movimiento, $\| l$ y $p_{\varphi}$, y dos grados de libcrlad $u$ y $\varphi$, así que cl movimiento es integrable. Las ecuaciones (2.3) y (2.4) $\operatorname{con} \lambda=0$ se reducen a:

$$
\begin{aligned}
\varphi^{\prime} & =\frac{2 \gamma_{1}}{u^{2}}-\frac{1}{u^{3}} \\
u^{\prime} u^{2} & = \pm\left(u^{4}-4 \gamma_{1}^{2} u^{2}+4 \gamma_{1} u-1\right)^{1 / 2}
\end{aligned}
$$

donde se ha usado que $p_{u}=u^{t}$ y $p_{\lambda}=u^{2} \lambda^{\prime}=0$.

Puede verificarse fácilmente que una solución de estas ecuaciones es $u=1$, $\gamma_{1}=1$ y $\varphi^{\prime}=1$ que es una órbita periódica circular. Esta órbita es inestable debido a que para $\gamma_{1}=1$, el potencial tiene un punto silla en $u=1$ (ver capitulo 3). Graef y Kusalia [16] encontraron la solución explícita para el caso ecuatorial en términos de funciones elípticas. Ellos demostraron que hay una región acotada de moviniento únicamente para $\gamma_{1} \geq 1$, y*que en esta región existen un conjunto numerable de valores de $\gamma_{1}>1$ para los que existen órbilas periódicas cerradas, mientras que para todos los otros valores de $\gamma_{1}>1$ el inovimiento es cuasiperiódico.

La ecuación diferencial de la trayectoria en el ecuador se obtiene climi- 
nando $s$ de (2.10) y (2.11):

$$
\left(\frac{d u}{d \varphi}\right)\left(2 \gamma_{1}-1 / u\right)= \pm \sqrt{u^{4}-4 \gamma_{1} u^{2}+4 \gamma_{1} u-1}
$$

y por lo tauto:

$$
\varphi= \pm \int \frac{\left(2 \gamma_{1}-1 / u\right) d u}{\sqrt{u^{4}-4 \gamma_{1}^{2}+4 \gamma_{1} u-1}}+c
$$

donde los dos signos se debe a que la trayectoria es simétrica respecto a una linea radial. Restringiendo la atención a la rama de signo positivo, la integra] en (2.12) se simplifica introduciendo la variable $y$ definida por:

$$
y^{2}=1+2 \gamma_{1} / u-1 / u^{2}
$$

es decir

$$
u^{-1}=\left(\gamma_{1}\right) \pm \sqrt{\gamma_{1}^{2}+1-y^{2}}
$$

La ecuación (2.12) queda:

$$
\varphi=\sin ^{-1}\left(\frac{y}{\sqrt{2}}\right) \mp\left(\frac{\gamma_{1}}{\sqrt{2}}\right)\left(\frac{1}{\sqrt{1+\gamma_{1}^{2}}}\right) I+c
$$

donde

$$
I=\int \frac{d y}{\sqrt{\left(1-y^{2} / 2\right)\left(1-y^{2} /\left(1+\gamma_{1}{ }^{2}\right)\right)}}
$$

El signo de (2.14) está delerminado por la elección de signo en (2.13).

Consideraré por separado los tres casos: $\gamma_{1}<1, \gamma_{1}=1$ y $\gamma_{1}>1$.

1. $\gamma_{1}<1$

Poniendo

$$
\frac{y}{\sqrt{1+\gamma_{1}^{2}}}=\sin \psi
$$

entonces

$$
I=\sqrt{1+\gamma^{2}} F(\alpha, \psi)
$$

donde $F$ es la integral elíptica de primera clase $y$

$$
\sin \alpha=k=\sqrt{\frac{1+\gamma_{1}^{2}}{2}}
$$


Entonces (2.13) y (2.14) resultan

$$
\varphi=\sin ^{-1}(k \sin \psi / \eta) \mp \frac{\gamma_{1}}{\sqrt{2}} r(\alpha, \psi)+c
$$

y

$$
u=\left(\gamma_{1} \pm \sqrt{1+\gamma_{1}^{2}} \cos \psi\right)^{-1}
$$

Las ecuaciones (2.15) y (2.16) son las ecuaciones parametricas de la trayectoria.

De la ccuación (2.16) vemos que $u$ cs no acotada y que la trayectoria. tiene dos dirccciones asintóticas:

$$
\psi=\cos ^{-1}\left(\frac{\gamma_{1}}{\sqrt{1+\gamma_{1}^{2}}}\right)
$$

y

$$
\psi=2 \pi-\cos ^{-1}\left(\frac{\gamma_{1}}{\sqrt{1+\gamma_{1}^{2}}}\right)
$$

2. $\gamma_{1}=1$

En este caso $I$ toma la forma:

$$
I=\int \frac{\mathrm{d} y}{1-y^{2} / 2}=\frac{1}{\sqrt{2}} \ln \left(\frac{\sqrt{2}+y}{\sqrt{2}-y}\right)
$$

$y$ de $(2.14)$ y (2.13)

$$
\varphi=\sin ^{-1} \frac{y}{\sqrt{2}} \mp \frac{1}{2 \sqrt{2}} \ln \left(\frac{\sqrt{2}+y}{\sqrt{2}-y}\right)+c
$$

y

$$
u=\left(1 \pm \sqrt{2-y^{2}}\right)^{-1}
$$

Obsérvese que cuando $u \rightarrow 1, y \rightarrow \sqrt{2}$ y $\varphi \rightarrow \infty$. Puesio que la velocidad es constante, a la partícula lc toma un tiempo infinito alcanzar el círculo unidad. La órbita no acotada (cxterna al círculo) es asintótica al círculo unidad y a una línea recta. 


\section{3. $\gamma_{1}>1$}

Ponicndo $y=\sqrt{2} \sin \psi$ sc obtiene:

$$
I=\sqrt{2} F(\alpha, \psi)
$$

donde

$$
\sin \alpha=\sqrt{2 /\left(1+\gamma_{1}^{2}\right)}
$$

Por lo tanto $(2.13)$ y $(2.14)$ resultan

$$
\begin{aligned}
u^{-1} & =\left(\gamma_{1} \pm \sqrt{\gamma_{1}^{2}+1-2 \sin ^{2} \psi}\right) \\
\varphi & =\psi \mp \frac{\gamma_{1}}{\sqrt{1+\gamma_{1}^{2}}} F(\alpha, \psi)+c
\end{aligned}
$$

El movimiento tendrá lugar en una de dos zonas scparadas. Una acotada por:

$$
\frac{1}{\left(\gamma_{1}+\sqrt{\gamma_{1}^{2}+1}\right)^{-1}} \leq u \leq \frac{1}{\left(\gamma_{1}+\sqrt{\gamma_{1}^{2}-1}\right)^{-1}}
$$

y otra no acotada:

$$
u \geq \frac{1}{\left(\gamma_{1}-\sqrt{\gamma_{1}^{2}-1}\right)^{-1}}
$$

En la zona acotada podrá haljer movimiento periódico y cuasiperiódico. Para que exista una órbita periódica, los períodos en $u$ y $\varphi$ deben ser conncusurables. De (2.19) y (2.21) se obtiene que

$$
\left(\frac{\gamma_{1}}{\sqrt{1+\gamma_{1}^{2}}} F(\alpha, \pi)-\pi\right) / 2 \pi=h\left(\gamma_{1}\right)
$$

asi que si $h\left(\gamma_{1}\right)=p / q$, con $p, q$ numeros enteros primos entre sí, la órbita tendrá $q$ rizos y se cerrará después de $p$ vueltas. P'uesto que $h$ es una función monótona decreciente de $\gamma_{1}[16]$ (ver figura (2.3)), cutonces los valores de $\gamma_{1}$ que corresponden a órbitas periódicas forman un conjunto numerabe. En la figura $(2.4)^{1}$ se muestra una trayectoria periódica con $p / q=1 / 5$.

\footnotetext{
${ }^{1}$ La figura (2.4) se obtuvo utilizando algunas identidades de las funciones elipticas y un método iterativo de funciones racionales desarrollado por el Dr. E. Piña.
} 


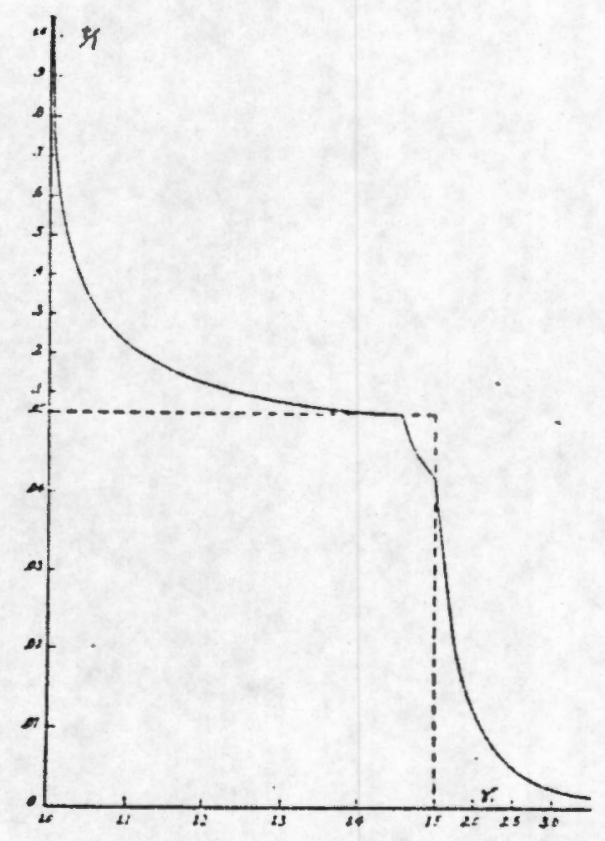

Figura 2.3: Gráfica de la función $h\left(\gamma_{1}\right)$

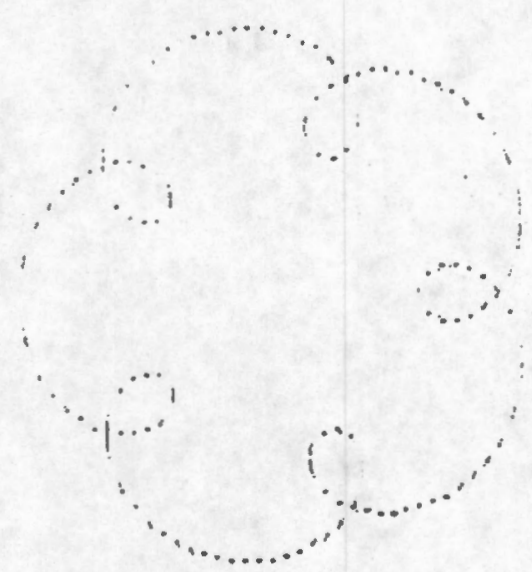

Figura 2.4: Órbita periódica con $p / q=1 / 5$, en $\gamma_{1}=1.03768$ 


\subsection{Ecuaciones de movimiento en el plano meridiano}

Si se supone quc el IIamiltoniano cn (2.3) depende únicamente de las variables $(u, \lambda)$ (jue defincn el plano meridiano, habrá que resolver un problena con dos grados de libertad $y$ una constante de movimiento: II. En lo gue sigue, enfocaremos la atención al movimiento en el plano meridjano. Debe tenerse en mente, sin cubargo, que una órbjta que es periódica cn el plano meridiano, generalmente no lo scrá en el espacio tridimensional.

Las ecuaciones de movimiento en el plano meridiano (2.5) y (2.6) no son integrables en términos de funciones conocilas (excepto en el caso $\lambda=0$ antes mencionado). Puesto que utilizaré mélodlos numéricos para resolverla, será convenicnte electuar una últinna trasformación debida a Störmer:

$$
\begin{aligned}
u & =\frac{1}{2 \gamma_{1}} c^{x} \\
\lambda & =\lambda \\
d \sigma & =8 \gamma_{1}{ }^{3} e^{-2 x} d s
\end{aligned}
$$

con el nuevo Hamiltoniano:

$$
E=\frac{1}{2}\left[p_{x}^{2}+p_{\lambda}{ }^{2}\right]-\frac{a c^{2 x}}{2}+\frac{1}{2}\left(\frac{1}{\cos (\lambda)}-e^{-x} \cos (\lambda)\right)^{2}=0
$$

donde $\dot{x}=p_{x}, \dot{\lambda}=p_{\text {入 }}$ y $a=1 /\left(2 \gamma_{1}\right)^{4}$. Aquí un punto sobre una letra significa derivada respecto de $\sigma$. Esta es una transformación conforme de Goursat que cambia coordenadas polares $(r, \lambda)$ en el plano meridiano a coordenadas cartesianas $(x, \lambda)$, y tiene la ventaja de sacar la singularidad $u=0$ de la. región finjta. Las ecuaciones de movimiento resultan:

$$
\begin{aligned}
& \ddot{x}=a e^{2 x}-e^{-x}+e^{-2 x} \cos ^{2}(\lambda) \\
& \vec{\lambda}=\tan (\lambda)\left[-1-\tan ^{2}(\lambda)+\cos ^{2}(\lambda) c^{-2 x}\right]
\end{aligned}
$$

con la integral de movimiento (2.25). 


\section{Capítulo 3}

\section{Curvas de velocidad cero y descripción cualitativa del movimiento}

El Hamiltoniano (2.3) describe el movimiento de una partícula en el plano meridiano con $p_{\varphi}=2 \gamma_{1}$ un parámetro. Éste se puede escribir como $H=$ $T+V$ donde el potencial es

$$
V=\frac{1}{2}\left(\frac{2 \gamma_{1}}{u \cos (\lambda)}-\frac{\cos (\lambda)}{u^{2}}\right)^{2}
$$

Calculando la derivada direccional de $V$ en la dirección de $u$, se obtiene:

$$
u \cdot \nabla V=\left(\frac{2 \gamma_{1}}{u \cos \lambda}-\frac{\cos \lambda}{u^{2}}\right)\left(\frac{-2 \gamma_{1}}{u^{2} \cos \lambda}+\frac{2 \cos \lambda}{u^{3}}\right)
$$

que corresponde a una fuerza radial repulsiva (dada por $-\mathbf{u} \cdot \nabla V>0$ ) si $\gamma_{1}<0$, así que no pueden existir órbitas atrapadas ni periódicas. En lo sucesivo consideraré sólo valores positivos de $\gamma_{1}$.

Obsérvese también que $V$ es no negativa y que toma su valor mínimo $V=0$ cuando

$$
\frac{2 \gamma_{1}}{u \cos \lambda}-\frac{\cos \lambda}{u^{2}}=0
$$

es decir, a lo largo de la línea de puntos críticos

$$
2 \gamma_{1} u=\cos ^{2} \lambda
$$


que es también la ecuación de una línea de campo y a la que se le denomina el "thalweg" descle Störmer. Cuando $\gamma_{1}=1$, hay además un punto de equilibrio aislado en $u=1, \lambda=0(\dot{o} x=\log 2, \lambda=0)$.

La partícula estará restringida a moverse en la región $0 \leq V \leq H$. Esta. región tendrá tres formas dependiendo de si $\gamma_{1}$ es monor que, igual a o mayor que 1.

Para analizar ésto, calculemos las curvas de velocidad cero haciendo ' $T$ ' $=0$ o $V=1 / 2$ :

$$
\left(\frac{2 \gamma_{i}}{u \cos \lambda}-\frac{\cos \lambda}{u^{2}}\right)^{2}=1
$$

entonces

$$
2 \gamma_{1} u / \cos \lambda-\cos \lambda=c u^{2}
$$

donde $c= \pm 1$. Si defino $w=2 \gamma_{1} u$ y $b=1 /\left(2 \gamma_{1}\right)^{2}$ entonces:

$$
c b w^{2}-w / \cos (\lambda)+\cos (\lambda)=0
$$

Esta ecuación tiene cuatro soluciones, poro una de cllas no tiene significado físico pues dá $w<0$ (es decir $u<0$ ). Las otras 3 son

$$
\begin{aligned}
& w_{1}=\frac{1 / \cos (\lambda)-\sqrt{1 / \cos ^{2}(\lambda)+4 b \cos (\lambda)}}{-2 b} \\
& w_{2}=\frac{1 / \cos (\lambda)-\sqrt{1 / \cos ^{2}(\lambda)-4 b \cos (\lambda)}}{2 b} \\
& w_{3}=\frac{1 / \cos (\lambda)+\sqrt{1 / \cos ^{2}(\lambda)-4 b \cos (\lambda)}}{2 b}
\end{aligned}
$$

El radical en $w_{1}$ es positivo cuando $\lambda \in(-\pi / 2, \pi / 2)$. El radical cn $w_{2}$ y $w_{3}$ será posilivo si $1 / 4 b>\cos ^{3}(\lambda)$. Cuando $b \geq 1 / 4$ (ie. $\gamma_{1} \leq 1$ ), las curvas $w_{2}$ y $w_{3}$ se tocan en un punto, y cuando $b<1 / 4$, no se tocan en uingun punto y resultan dos zonas disconexas.

En coordenadas cilindricas

$$
\begin{aligned}
& \rho=u \cos \lambda \\
& z=u \sin \lambda
\end{aligned}
$$




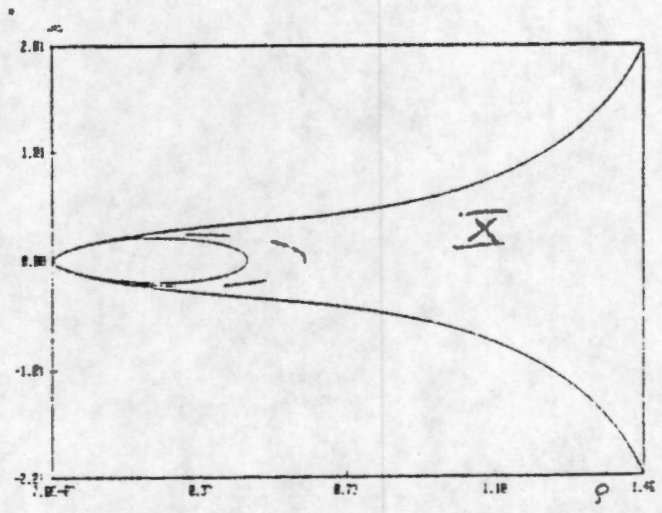

Figura 3.1: Región de Hill para $\gamma_{1}=0.5$ (marcada con $X$ ). El thalweg se muestra con linca interrumpida.

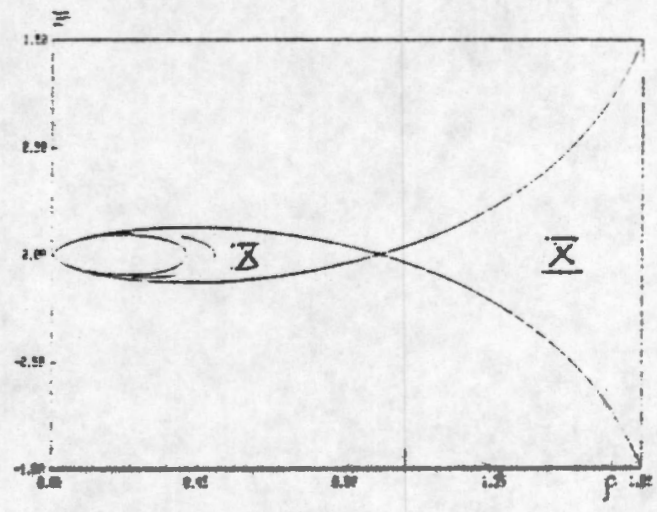

Figura 3.2: Región de Hill para $\gamma_{1}=1.0$.

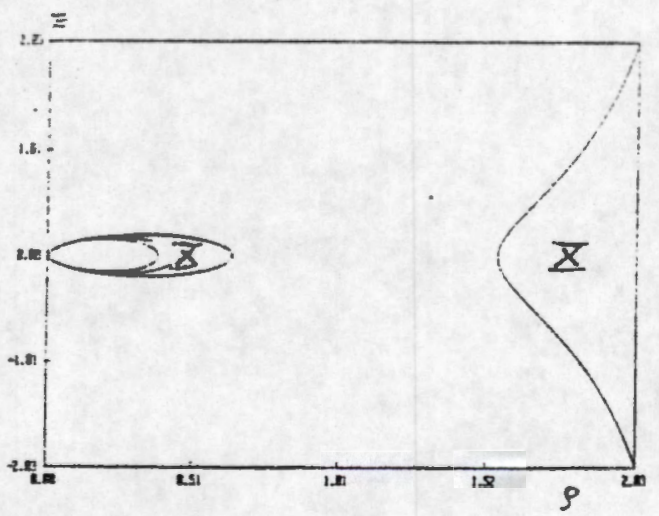

Figura 3.3: Región de Hill para $\gamma_{1}=1.1$. 
las ecuaciones (3.4) resultan

$$
\begin{aligned}
& w_{1}=\left(u / \rho-\sqrt{u^{2} / \rho^{2}+4 b \rho / u}\right) /(-2 b) \\
& w_{2}=\left(u / \rho-\sqrt{u^{2} / \rho^{2}-4 b \rho / u}\right) /(-2 b) \\
& w_{1}=\left(u / \rho+\sqrt{u^{2} / \rho^{2}-4 b \rho / u}\right) /(-2 b)
\end{aligned}
$$

donde $u=\sqrt{\rho^{2}+z^{2}}$. Estas coordenadas las utilizaré para graficar las trayectorias en cl espacio de configuración (plano meridiano).

En las figuras (3.1) a (3.3) mucstro curvas típicas correspondientes a $\gamma_{1}<1, \gamma_{1}=1$ y $\gamma_{1}>1$ respectivanente en el plano meridiano $(\rho, \approx)$ (o $(u, \lambda))$. Ja region permitida $V \leq H$ es la llamada región de Ilill, es decir, la región en el espacio de configuración donde el movimiento está permitido.

En cl caso en unue $\gamma_{1} \gg 1$, la partícula estará alrapada cerca del mínimo del potencial y oscilará alrededor del thalweg, ec. (3.2). Braun [5] cstudió este caso, y dcmostró varios hechos que intuitivamcnte pueden esperarse. La. carga se mueve entre dos puntos espejo cn laclos opuestos al plano ecuatorial. Puesto que su velocidad $v$ es constante, cuando la carga se mueve a regiones de latilud elevada, la magnilud del campo magnético y la frecuencia de ciclotrón se elevan mientras que el radio de giro disminuye así que la. partícula gira rápidamente en un movinniento oscilatorio alrededor del thatweg. En ésta aproximación, Braun muestra que los cruces consecutivos de la trayectoria con el thalweg forman una sucesión monótona que tiende a $\pi / 2$ conforme aumenta la latitud. Cuando la carga regresa hacia el ecuador, estos ángulos de cruce decrecen monótonamente (figura (3.4)). El resultado más importante al que él llega es que existen movimientos cuasipcriódicos (toros invariantes) muy próximos al thalweg (o línea de campo) que pueden acercarse al dipolo tanto como se quiera, y que atrapan a la carga permanentemente. Un corolario de ésto es que deben existir infinitas soluciones periódicas en la zona acotada de la región de Hill cuando $\gamma_{1} \gg 1$.

Otra cucstión de mucho interés ha sido la existencia y unicidad (para. cada valor del parametro $\gamma_{1}$ ) de una órbita que entre al dipolo. Störwer [41] encontró un desarrollo formal en series que, si converge, representaría una trayectoria que llega a la singularidad $u=0, \lambda= \pm \pi / 2$ (ver capítulo 5). Con este desarrollo, aunado a una gran cantidad de cálculos numéricos, él conjeturó la existencia y unicidad de una órbita que entra al dipolo. Malnquist [26] probó la existencia de al menos una de tales soluciones cuyo desarrollo 


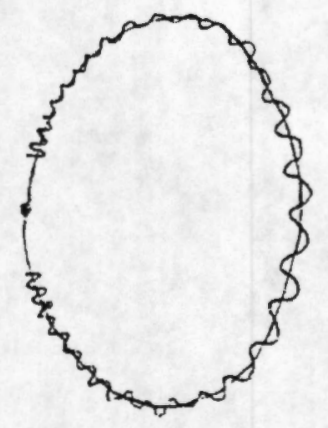

Figura 3.4: 'Traycctoria de la carga para $\gamma_{1} \gg 1$. La carga oscila rápjdamente adrededor de thalweg y rebota entre dos puntos espejo cn lados opuestos al ecuador.

asintótico Fur dado por la seric formal de Störmer. Finalmente, Braun [6] dió una demostración altcrnativa para la existencia de una órbita que entra al dipolo y una que sale de él, y luego mostró su unicidad. 


\section{Capítulo 4}

\section{Sección de Poincaré y líneas de simetría}

Mi objetjo es estudiar las órbitas periódicas en el plano meridiano, cuyas ecuacioncs de movimiento están dadas por la ecuación (2.26) con la cunstante de movimiento (2.25). Para ello, introduzco la sección de Poincaré en el plano $\lambda=0$ (ecuatorial) que denotaré por $\wp$, intersectando la trayectoria fase de coordenadas $x_{1}=x, x_{2}=\lambda, x_{3}=\dot{x}$ y $x_{4}=\dot{\lambda}$ con el plano fr. Cada. intersección del punto fase con el plano de Poincaré deja un punto en 8 .

Un punto $\mathrm{x}=\left(x_{1}, x_{3}\right)$ en $\wp$ es suficiente para definir las condiciones iniciales de la órbita en el espacio fase puesto que $x_{2}=0$ y $x_{4}$ se deriva de la ecuación (2.25) salvo un signo. Escogeré el signo positivo de $x_{4}$ en las condiciones iniciales.

Sea $\mathrm{x}$ un punto en el plano de Poincaré $\wp$, y sean $T \mathbf{x}, T^{2} \mathbf{x}, \ldots$, elc.las intersecciones sucesivas de la trayectoria fase con $\wp$. $T^{\prime}$ es el operador de evolución temporal y es una transformación canónica. Concretamente, aplicar $T$ a un punto en 87 significa integrar numéricamente las ecuaciones diferenciales (2.26) con cse punto como condiciones iniciales, hasta encontrar el siguiente punto de cruce con el plano $\wp$.

El problema de cncontrar soluciones periódicas de las ecuaciones diferenciales (2.26) es cquivalente a determinar los puntos fijos de la transformación $T$ del plano $\lambda=0$ en sí mismo.

Si $n$ es el entero positivo más pequeño tal que $T^{n} \mathbf{x}=\mathbf{x}$, se dice que el conjunto $O=\left\{\mathbf{x}, T \mathbf{x}, \ldots, T^{n-1} \mathbf{x}\right\}$ es una órbita periódica de período $n$ en el plano de Poincaré. 
Si se paramctriza la matríz de Flocpuet $L$ de la órbita periódica en la forma de Cayley-Klein [36]:

$$
L=\left(\begin{array}{cc}
a+d & c+b \\
c-b & a-d
\end{array}\right)
$$

con la condición de que se preserve el área dada por:

$$
a^{2}+b^{2}-c^{2}-d^{2}=1
$$

se tendráu tres casos para los exponentes característicos de Floquet:

1. Estable ó elíptico: Los autovalores de $L$ son complejos conjugados

$$
a=\exp ( \pm i A)
$$

y el paránnetro $a=(1 / 2) l r L$ se puede expresar en térninos del ángulo $A$ como $a=\cos A$.

2. Inestable o hiperbólico: Los autovalores de $L$ son reales y diferentes

$$
\alpha=\epsilon \exp ( \pm A) \quad(\epsilon=1 \quad 0 \quad-1)
$$

y $a=c \cosh A$.

3. Parabólico: Los autovalores de $L$ son reales, iguales y de maguitud 1

$$
\alpha=\epsilon=a
$$

Introduciendo otros dos parámetros reales $B$ y $C$ por

$$
\begin{aligned}
& b=\sin A \cosh B \\
& c=\sin A \sinh B \sin C \\
& d=\sin A \sinh B \cos C
\end{aligned}
$$

$L(A, B, C)$ es una representación del grupo de rotación pues

$$
L^{\mathfrak{q}}(A, B, C)=L(q A, B, C)
$$

Considerando en 4.1 los valores de $q$ y $p$ tal que

$$
q A=2 \pi p \quad(p<q ; \quad p, q \quad \text { coprimos })
$$


entonces la polencia $q$ de $L$ resulla la matríz unidad

$$
L^{q}(A, B, C)=L(2 \pi, B, C)=1
$$

Así, $\Lambda$ es el ásigulo promedio que rotan los puntos vecinos al punto periódico (de período $q$ ), cuando los puntos vecinos se transforman mediante la matríz lincarizada $L$ de $T^{q q}$ en el punto periódico.

Cuando la transformación $T$ se pucde escribir como el producto de dos involuciones:

$$
T=I_{1} I_{0}
$$

donde $I_{1} I_{1}=I_{0} I_{0}=1$ (ic. $I_{0}$ e $l_{1}$ son involuciones), se dice que $I$ cs reversible. Este término es una gencralización de un caso que se encuentra frecuenlemente en la física: si la transformación 'I' está determinada por un Hamiltoniano cuadrático $\mathrm{cn}$ las velocidades, cutonces $I_{0} \mathrm{c} I_{1}=T I_{0}$ son la inversión en la velocidad y en el ticmpo respectivament.c, pues $T^{-1}=I_{0} T I_{0}$, y el sistema es reversible.

A $T$ se le llama una transformación sinćtrica respecto de $J_{0}$.

El problema de Störmer tiene la simetría.

$$
\left(x_{1}, x_{2}, x_{3}, x_{4}\right) \rightarrow\left(x_{1},-x_{2},-x_{3}, x_{4}\right)
$$

o en el plano de Poincaré:

$$
I_{0}\left(x_{1}, x_{3}\right)=\left(x_{1},-x_{3}\right)
$$

$I_{0}$ es una involución pues

$$
I_{0} I_{0}=1
$$

La factorización de $T$ en el producto de dos involuciones fué introducida por Birkhof [2], [3] y ampliamente estudiada por De Vogelaerc [11]. Si $T$ ' se factoriza, se pucde definir la transformación $I_{j}$ para $j$ un entero arbitrario por:

$$
I_{j}=T^{j} I_{0}
$$

que también es una involución pues:

$$
J_{j} I_{j}=1
$$

Nótese que $T^{-1}=I_{0} I_{1}$ pues $T\left(I_{0} I_{1}\right)=I_{1} I_{0} I_{0} I_{1}=1$ y análogamente $\left(I_{0} I_{1}\right) T=1$ 
El conjunto de transformaciones $\left\{I_{j}, T^{k}\right\}$, con $j$ y $k$ : en $Z$ (como es usual $Z$ es el conjunto de los cnteros), es un grupo infinito discreto [36] con la representación

$$
\begin{aligned}
I^{j} I_{k} & =I_{j+k} \\
J_{j} I_{k} & =T^{j-k} \\
I_{j} T^{k} & =I_{j-k}
\end{aligned}
$$

Se define ahora el subconjunto $\Gamma_{j}$ de $\gamma$ como el conjunto de puntos fijos bajo $I_{j}: \Gamma_{j}=\left\{\mathrm{x} \in \delta \mid I_{j} \mathrm{x}=\mathrm{x}\right\}$. $\Gamma_{j}$ es una línca que puede scr discontínua ${ }^{1}$ como en el problema restringido de tres cuerpos [7]. $\wedge \Gamma_{j}$ se le llama la línea de sinctria $j$ [36]. En el problema de Störmer las líneas de simetría son contínuas. Denotando además por $P_{n}$ al conjunto de puntos fijos bajo $T^{n}$ :

$$
P_{n}=\left\{\mathrm{x} \in \wp \mid T^{n} \mathrm{x}=\mathrm{x}\right\}
$$

se pueden demostrar algunas propiedades importantes, para $j, k, l y n$ enteros arbitrarios:

1. Si $\mathbf{x} \in \Gamma_{j} \cap \Gamma_{k} \Rightarrow T^{|j-k|} \mathbf{x}=\mathbf{x}$.

Esto se sigue directamente de que $I_{j} I_{k}=I^{j-k}$. Si $\mathrm{x} \in \mathrm{I}_{j} \cap \mathrm{I}_{k} \Rightarrow$ $I_{j} \mathrm{x}=\mathrm{x}$ y $I_{k} \mathrm{x}=\mathrm{x} \Rightarrow T^{j-k} \mathrm{x}=\mathrm{x} \Rightarrow \mathrm{x} \in P_{|j-k|}$.

Por lo tanto, si un punto está cu la intersección de dos líneas de simetría, digamos $\Gamma_{j}$ y $\Gamma_{k}$, entonces el punto $\mathrm{x}$ es periódico y su periodo divide a $|j-k|$.

2. Líneas de simetria se mapcan bajo $T$ ó $I$ en líneas de simetría:

$$
\begin{aligned}
T^{n} \Gamma_{k} & =\Gamma_{2 n+k} \\
I_{n} \Gamma_{k} & =\Gamma_{2 n-k}
\end{aligned}
$$

\section{Demostración}

Sea $\mathrm{x} \in \Gamma_{k} \Rightarrow I_{k} \mathrm{x}=\mathrm{x}=I_{n+k-n} \mathbf{x}$. Pero $I_{n+k-n} \mathrm{x}=7^{n+k} I_{-n} \mathrm{x}$ por la ecuación (4.4), y entonces $\mathbf{x}=T^{n+k} I_{-n} \mathbf{x}=T^{n+k} I_{0} T^{n} \mathbf{x}$. Aplicando $T^{n}$

\footnotetext{
${ }^{1}$ Condiciones para la continuidad de $\Gamma_{j}$ están dadas en [11] pag. 72
} 
en ambos lados: $T^{2 n+k} I_{0} T^{n} \mathrm{x}=T^{n} \mathrm{x}$. Entonces $I_{2 n+k} T^{n} \mathrm{x}=T^{n} \mathrm{x}$, es decir $T^{n} \mathrm{x} \in \Gamma_{2 n+k}$. Por lo tanto

$$
T^{n} \Gamma_{k} \subset \Gamma_{2 n+k}
$$

$\Lambda$ horn, sea $\mathrm{x} \in \Gamma_{2 n+k}$. Veré que $T^{-n} \mathrm{x} \in \Gamma_{k}$. Se tiene que $I_{2 n+k} \mathrm{x}=$ $\mathrm{x} \Rightarrow I_{n+k} T^{-n} \mathrm{x}=\mathrm{x}$ por (1.6), cutonces $T^{n+k} I_{0} T^{-n} \mathrm{x}=\mathrm{x}$. $\Lambda$ plicando $T^{-n}$ cn ambos lados: $T^{k} J_{0} T^{-n} \mathrm{x}=T^{-n} \mathrm{x}$, por lo tanto $T^{-n} \mathrm{x} \in \Gamma_{k}$, es decir:

$$
\Gamma_{2 n+k} \subset \eta^{m} \Gamma_{k}
$$

con lo que queda demostrado (1.8). Análogamente se demucstra (4.9). La ecuación (4.8) es muy útil pues permile calcular todas las líneas pares en base a la línea $\Gamma_{0}$, simplemente iterándola con $T$ ': $\Gamma_{2}=T I_{0}$, $\Gamma_{4}=T \Gamma_{2}, \ldots$ ctc. Similarmente, $\Gamma_{1}$ genera todas las lineas impares: $\Gamma_{3}=T \Gamma_{1}, \Gamma_{5}=T \Gamma_{3}, \ldots$ etc.

Como un caso particular de (4.9), se tiene la siguiente propiedad.

3. $I_{0} \Gamma_{n}=\Gamma_{-n}$

Esta propiedad dá una manera sencilla de calcular las líneas uegalivas.

4. Si $x \in \Gamma_{j} \cap \Gamma_{j+k}$ entonces $\mathrm{x} \in \Gamma_{j+l k}$ para todo $l \in Z$ (como es usual, $Z$ denota el conjunto de enteros).

DEMOSTHACión

Si $\mathbf{x} \in \Gamma_{j} \cap \Gamma_{j+k} \Rightarrow I_{j} \mathbf{x}=\mathbf{x}$ y $J_{j+k} \mathbf{x}=\mathbf{x}$. Por (4.5) se tienc que: $I_{j+k} I_{j} \mathrm{x}=T^{k} \mathrm{x}=\mathrm{x}$, es decir $\mathrm{x}$ es un punto fijo de $T^{k}$, aunque su periodo puede ser un divisor de $k$. Asi, $\eta^{l k} \mathrm{x}=\mathrm{x}$ para todo $l \in Z$.

Además, puesto que $x \in \Gamma_{j} \Rightarrow T^{j} I_{0} \mathrm{x}=\mathrm{x}$ y aplicando $T^{\text {lk }}$ cu ambos lados de la igualdad se obtiene:

$$
T^{i+l k} I_{0} \mathrm{x}=I_{j+l k} \mathrm{x}=T^{l k} \mathrm{x}=\mathrm{x}
$$

por lo tanto $\mathrm{x} \in \Gamma_{j+l k}$.

5. Sca $\mathrm{x}$ un punto de una órbita de período $k$ en el plano de Poincaré $\wp$. Entonces la órbita es invariante respecto de $I_{0}$ si y solo si x está en la. línea de simetria $\Gamma_{j}$, donde $0 \leq j \leq k-1$. 


\section{DEMOSTRACIÓN}

La órbita $O$ de $\mathrm{x}$ es invariaute respecto de $I_{0} \Leftrightarrow J_{0} \mathrm{x} \in O \Leftrightarrow \exists j \in Z$, $0 \leq j \leq k-1$ tal que $I_{0} \mathrm{x}=T^{-j} \mathrm{x} \Leftrightarrow T^{i} I_{0} \mathrm{x}=\mathbf{x}=I_{j} \mathbf{x}$ ic. $\mathrm{x} \in \Gamma_{j}$.

6. Sea $\mathrm{x} \in \mathrm{I}$ s un punto de una órbita de período $k$, con $0 \leq s \leq k-1$ y sea $t$ un entero arbitrario daclo. Entonces algun punto de la órbita. de $\mathrm{x}$ estará sobre la línca $\Gamma_{t}$, siendo la única restricción subre $t$ que $(t+k-s)$ sea par.

\section{Demostración}

Como $\mathrm{x} \in \Gamma_{s}, I_{s} \mathrm{x}=\mathrm{x}, y$ culonces $I_{s} \mathrm{x}=I_{t} T^{t-s} \mathrm{x} \forall t \in Z$ por (4.6). Aplicaudo $f_{t}$ en ambos larlos se obtiene:

$$
T^{1-s} \mathrm{x}=I_{1} \mathrm{x}
$$

Ahora, debido a (4.4),

$$
J_{t} \mathrm{x}=T^{(1+k-s) / 2} J_{(t-k+s) / 2} \mathrm{x}
$$

para $t \in Z$ tal que $(t+k-s)$ sea par.

Usando (4.6) en (6) se ticne que

$$
I_{t} \mathrm{x}=T^{(t+k-s) / 2} I_{i} T^{(t+k-s) / 2} \mathrm{x}
$$

y sustituyendo en (6):

$$
T^{t-s} \mathrm{x}=T^{(t+k-s) / 2} I_{t} T^{(t+k-s) / 2} \mathbf{x}
$$

Por otro lado, $T^{k} \mathrm{x}=\mathrm{x}$, así que la ecuación anterior resulla y puesto que $T^{k} \mathbf{x}=\mathbf{x}$, se tiene que:

$$
T^{t+k-s} \mathrm{x}=T^{(t+k-s) / 2} I_{t} T^{(t+k-s) / 2} \mathrm{x}
$$

y por lo lanto:

$$
T^{(t+k-s) / 2} \mathrm{X}=I_{t} T^{(t+k-s) / 2}
$$

es decir, $T^{(t+k-s) / 2} \mathrm{x} \in \Gamma_{t}$, donde $k$ es el período de la órbita de $\mathrm{x}, s$ es un entero tal que $0 \leq s \leq k-1$ y $t$ es cualquier entero tal que $(t+k-s)$ es par. Como corolario se tienen las tres siguientes propiedades. 
7. Supóngase que $x$ es un punto de una órbita invariante con respecto a $I_{0}$ en el plano de Poincaré y con periodo k. Entonces ocurre una y solo una de las siguientes posibilidades:

La órljita liene

(a) Jos puntos, y solo dos, sobre $\Gamma_{0}$ y ninguno sobre $\Gamma_{1}$ si $k$ es par.

(b) Dos puntos, y solo dos, sobre $l_{1}$ y uinguno sobre $\Gamma_{0}$ si $k$ es par.

(c) In único punto sobre $\Gamma_{0}$ y otro úuico punto sobre $\Gamma_{1}$ si $\mathrm{K}$ es impar (cxeplo en el caso $n=1$, donde el mismo punto está on $\Gamma_{0} \mathrm{~g}$ en $\left.l_{1}\right)$.

\section{Demostriación}

(a.) Supóngase que $k$ es par y que $\mathrm{x} \in \Gamma_{s}$, s par. Entonces $l+k-s$ es par sólo si $l$ es par o ccro, ic., por la órbila periódica de $x$ sólo pasan lincas pares o la cero. P'or lo tanto hay un punto $y=J^{k-s}$ de la orbila que está en $\mathrm{I}_{\mathrm{o}}: J_{\mathrm{0}} \mathrm{y}=\mathrm{y}, \mathrm{y}$ por (4.4)

$$
7^{k / 2} I_{-k / 2 y}=y=T^{k} y
$$

y aplicando $7^{-k / 2}$ resulta

$$
I_{-k / 2 y}=T^{k / 2} y
$$

y por (4.6)

$$
I_{0} T^{k / 2} \mathbf{y}=T^{k / 2} \mathbf{y}
$$

cs decir, $T^{k / 2} y \in \Gamma_{0}$ y la órbita ticne dos puntos en $\Gamma_{0}$ y ninguno en $\Gamma_{1}$ (ni en ninguna línea impar).

(b) Supóngase que $k$ es par y que $\mathrm{x} \in \Gamma_{s}, s$ impar. Entonces $t+k-s$ es par sólo si $t$ es impar, es decir, por la órbita periódica de $\mathbf{x}$ sólo pasan lincis impares. En parlicular, el punlo $y=7^{(1+k-s) / 2} \mathrm{x} \in$ $I_{1}: I_{1} y=$ y y por (1.1)

$$
T^{k / 2} I_{1-k / 2} \mathbf{y}=\mathbf{y}=T^{k} \mathbf{y}
$$

entonces

$$
I_{1-k / 2} \mathrm{y}=7^{k / 2} \mathrm{y}
$$


y aplicando (4.6)

$$
I_{1} T^{k / 2} \mathrm{y}=T^{k / 2} \mathrm{y}
$$

es decir, $T^{k / 2} y \in l_{1}^{\prime} y$ la órbila tiene dos puntos en $J_{1}$, y ninguno en $\Gamma_{0}$ (ni en ninguna línea par).

(c) Supóngase que $k$ es impar y que $\mathrm{x} \in \Gamma_{s}$.

Si s es par, culonces $t+k-s$ es par si $t$ es impar. Sea $y=$ $J^{1+k-s} \mathrm{x} \in \Gamma_{1}$, ie. $I_{1} \mathrm{y}=\mathrm{y} . \Lambda$ plicassdo $(1.6)$

$$
I_{(k+1) / 2} T^{(k-1) / 2} \mathbf{y}=\mathbf{y}
$$

y por (4.4)

$$
T^{(k+1) / 2} I_{0} T^{(k-1) / 2} \mathbf{y}=\mathbf{y}=T^{k} \mathbf{y}
$$

pucs $\mathrm{y}$ es de periorlo $k$. $\Lambda$ plicando $T^{-(k+1) / 2}$ se obtienc

$$
J_{0} T^{(k-1) / 2} y=T^{(k-1) / 2} y
$$

es decir, $T^{(k-1) / 2} y \in \Gamma_{1}$.

Análoganıente se demuestra que si $s$ es impar, hay un punto $\mathbf{y}=$ $T^{k-s} \mathrm{x} \in \mathrm{I}_{0}$ y otro punto $T^{(k+1) / 2} \mathrm{y} \in \Gamma_{1}$.

8. Por los puntos de período 1 simétricos respecto a $I_{0}$ pasan todas las lineas de simetría.

9. Las lineas $\Gamma_{n}$ y $\Gamma_{n+1}$ sólo se intersectan en puntos sobre $\Gamma_{0}$

Como ya se habia dicho, el problema de Störmer tiene la simetría:

$$
l_{u}\left(x_{1}, x_{3}\right)=\left(x_{1},-x_{3}\right)
$$

de manera que $\Gamma_{0}$ es la línea $x_{3}=0$.

Por otro lado, si un punto fase $\left(x_{1}, x_{2}=0, x_{3}, x_{4}\right) \in \wp$ al liempo $t=0$ evoluciona con cl flujo al punto $\left(x_{1}, x_{2}=0,-x_{3},-x_{4}\right) \in \wp$ al tiempo $t=\tau$, ie. $T \mathbf{x}=I^{\prime}\left(x_{1}, x_{3}\right)=\left(x_{1},-x_{3}\right)$, cntonces en $t=\tau / 2$ el punto fase toca la. curva de velocidad cero, y la partícula regresará por la misma trayectoria con velocidad opuesta. Nótese que $\mathrm{x} \in \Gamma_{-1}$ pues $I_{-1} \mathrm{x}=I_{0} T \mathrm{x}=I_{0}\left(x_{1},-x_{3}\right)=$ $\left(x_{1}, x_{3}\right)=x$. Se sigue también que $T x \in \Gamma_{1}$.

Por lo tanto, para calcular $\Gamma_{1}$, simplemente se toman puntos con condiciones iniciales sobre la curva de velocidad cero y se encuentra su primer 
cruce con la sección de Poincaré. Las otras líneas se calculan utilizando las propiedades 2 y 3 . La propiedad 8 se muestra en forma gráfica en la sección 5.1.

Se utilizó un método Runge Kutta orden 4 para integrar las ecuaciones de movimiento (2.26) y calcular cl mapeo $T$ de Poincaré.

- Las órbilas de período impar $n$ se deben iterar $2 n$ veces para obtener la órbita periódica completa en el espacio fase: cuando la órbita se itera $n$ veces, regresa al mismo punto cn el plano de Poincaré pero con $x_{4}$ de signo opuesto al original (pues $n$ es impar). Estas órbitas periódicas son trayectorias también simétricas en cl espacio de configuración pues tiencu un punto $\mathrm{x} \in \mathrm{I}_{\mathrm{u}}$ donde la particula cruza perpendicularmente al ecuador, $\mathrm{y}$ otro punto $T^{(n+1) / 2} x \in \Gamma_{1}$, asi (jue entre $T^{(n-1) / 2} x$ y $T^{(n+1) / 2} x$ lat trayecturia de la partícula toca la curva de velocidad cero y regresa sobre sí misma produciendo un extremo abierto de la trayectoria. Al iterarse $2 n$ veces, loa trayectoria se recorre cu sentido opuesto, produciendo el otro extremo abierto simétrico al primero. Por lo tanto, las órbitas de período impar son abiertas [30] y simétricas en el espacio de configuración.

Las órbitas de período par $n$ con dos puntos $x \in \Gamma_{0}$ y $T^{n / 2} x \in \Gamma_{0}$, cruzan en esos puntos al ecuador perpendicularmente, así que son cerradas y simétricas cu cl espacio de configuración.

Las órbitas de periodo par $n$ con dos puntos $x \in \Gamma_{1}$ y $T^{n / 2} x \in \Gamma_{1}$ locan la curva de velocidad cero en dos puntos no simétricos en el espacio de configuración. Puesto que no ticuen ningún punto en $\Gamma_{0}$, nunca cruzan al ecuador perpendicularmente. Son órbilas abiertas y asimétricas en el espacio de configuración, aunque son simétricas respecto a $I_{0}$ en el plano de Poincaré.

En lo sucesivo, cuando se diga que una órbita es simétrica 0 asimétrica se entenderá que lo es en el espacio de configuración $(u, \lambda)$ : coordena.das polares (en el plano meridiano); $(\rho, z)$ : coordenadas cartesianas.

Finalmente, puesto que $E=0$ y $x_{4}{ }^{2} \geq 0$ en la ec (2.25), la región permitida en el plano de Poincaré es:

$$
\left|x_{3}\right| \leq \sqrt{a e^{2 x_{3}}-\left(1-e^{-x_{1}}\right)^{2}}
$$

Todas las líneas de simetría deben de estar en el interior de esta región. Formas típicas se muestran en las figuras (4.1) a (4.3) para $\gamma_{1}<1, \gamma_{1}=1$ y $\gamma_{1}>1$ respectivamente.

La frontera de la región permitida (cuando se cumple la igualdad en la 


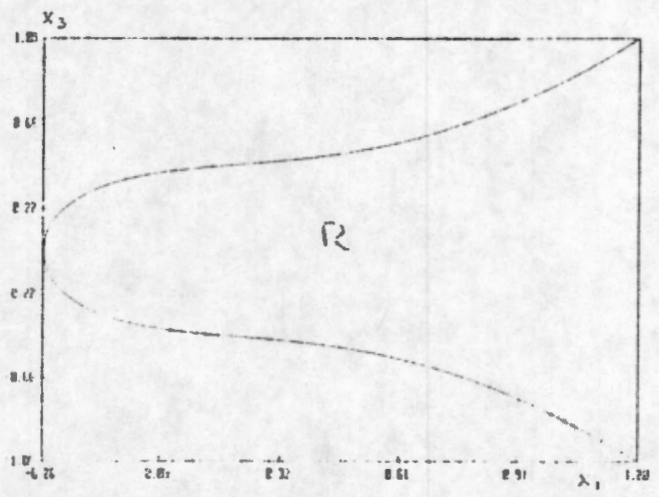

Figura 4.1: $R$ es la región permitida en el plajo de Poincaré para $\gamma_{1}=0.8$

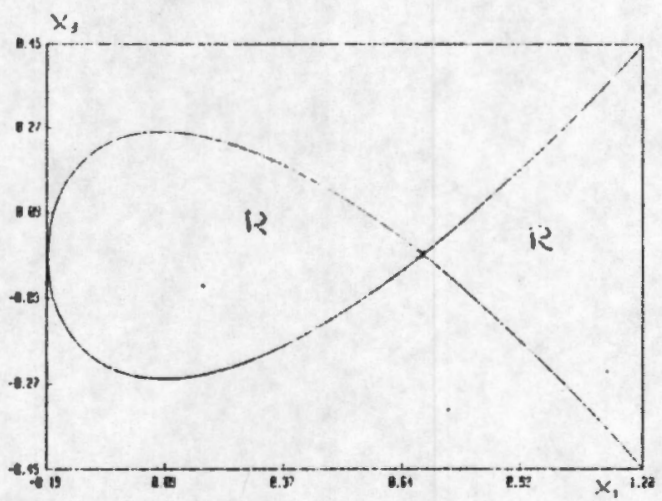

Figura 4.2: $R$ es la región permilida en el plano de Poincaré para $\gamma_{1}=1.0$

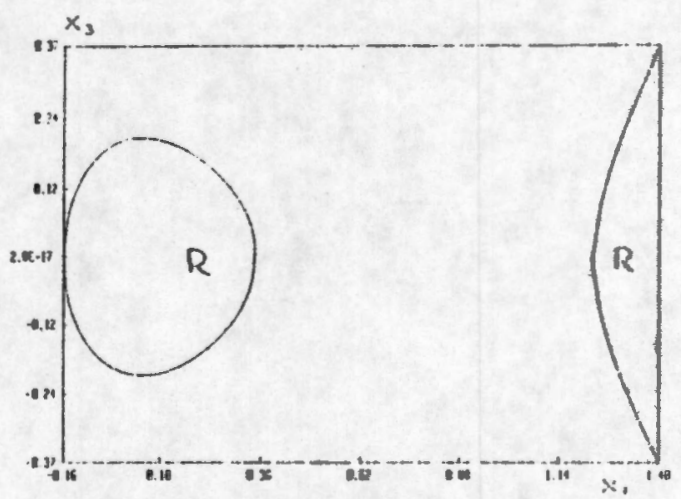

Figura 4.3: $R$ es la región permitida en el plano de Poincaré para $\gamma_{1}=1.1$ 
ccuación (1.13)) en (y se denotará por E y represcnta movimiento ecuatorial pues $\lambda=0$ y $x_{1}=p_{\lambda}=0$. 


\section{Capítulo 5}

\section{Órbitas periódicas}

Störmer [40] encontró que había una infinjdad de movimientos periódicos en el plano meridiano, entre los que cabe distinguir las órbitas que tienen la propiedad de intersectar al ecuador en ángulos rectos (órbitas simétricas). Sus cálculos numéricos le dieron una estimación de que estas órbitas deberían existir para $\gamma_{1}>0.3535$, refinándolo después a $\gamma_{1}>0.5$.

Lemaitre y Vallarta [24] demostraron que no podían existir órbitas periódicas para $\gamma_{1}<0.783$. También encontraron que la llamada órbita "principal" externa (ver sección 5.1) sólo existía en el rango $0.783<\gamma_{1}<1$. El límite inferior fué refinado posteriormente por Lemaitre [25] a 0.78856 . $\mathrm{El}$ valor $\gamma_{1}=1$ corresponde a la órbita circular en el plano ecuatorial. Finalmente, Godart [14] demostró la existencia de soluciones periódicas para $\gamma_{1}>\gamma_{1}{ }^{*}=0.788541$.

En lo que sigue presentaré un análisis de las líneas de simetría y órbitas periódicas cuando el parámetro de Störmer $\gamma_{1}$ cambia.

Se relaciona también la forma de las órbitas periódicas con el estudio de regiones hecho por Graef [17].

En la primera sección se calculan las líneas $\Gamma_{-6}, \ldots, \Gamma_{0}, \ldots, \Gamma_{6}$ para algunos valores de $\gamma_{1}$ y se estudia la llamada familia principal $f_{0}$ de órbitas periódicas. Como se verá, muchas de las propiedades presentadas por Piña et. al. [36] se aplican a este problema. Así mismo, muchos de los resultados conocidos para el problema de Störmer y sus órbitas periódicas se obtienen de manera natural en base al estudio de las líneas de simetría. Se hace una correspondencia con los estudios del grupo de investigadores griegos [28]-[31]. Se muestran las líneas de simetría cuando $\gamma_{1}$ es cercano a $\gamma_{1}{ }^{*}$. Después se 
presenta el nacimiento de la familia principal $f_{0}$ y sus bifurcaciones. Se verá que la tangencia entre líneas de simetría indica un punto de bifurcación.

En la segunda y tercera sección se enfoca el análisis a dos líneas particulares: $\Gamma_{1}$ y $\Gamma_{2}$. El seguimiento de estas líneas al cambiar el parámetro $\gamma_{1}$ dá la estructura global de las llamadas órbitas periódicas simples ${ }^{1}$ :

1. De período 1 .- Son órbitas simétricas abiertas denotadas por $f_{0}, f_{2}, \ldots$, $f_{2 n}$.

2. De período 2 con dos puntos sobre $\Gamma_{0}$.- Son órbitas simétricas cerradas denotadas por $f_{1}, f_{3}, \ldots, f_{2 n+3}$.

3. De periodo 2 con dos puntos sobre $\Gamma_{1}$.- Son órbitas asimétricas abiertas denotadas por $a_{1, l}, a_{2, l}, \ldots, a_{n, l}(l \in Z)$.

En el siguiente capitulo se estudiarán las órbitas de periodo 3 .

\subsection{La familia principal}

Se calcularon varias líneas de simetría para diferentes valores del parámetro (ver figuras de este capítulo y el siguiente).

$\Gamma_{1}$ está formada por dos ramas espirales que corresponden a las dos ramas de la curva de velocidad cero que convergen a la singularidad $u=0$, $\lambda= \pm \pi / 2$. Malmquist [26] demostró que existe al menos una órbita que va a la singularidad para cada valor fijo de $\gamma_{1}$, aunque la demostración de unicidad fué dada posteriormente por Braun [4]. Esto es importante pues si únicamente una trayectoria $\tau$ llega a la singularidad y si se considera a la trayectoria que "sale" de la singularidad como la trayectoria $\tau$ recorrida en sentido opuesto, las dos espirales de $\Gamma_{1}$ convergen a un punto que denoto por $\xi_{1}$ en en el plano $p$. Este punto es la primera intersección con el plano de Poincaré de la trayectoria que sale de la singularidad y que puede ser considerado como un punto de $\Gamma_{1}$ si se define $T^{-1} \xi_{1}=I_{0} \xi_{1}$.

Puesto que ninguna trayectoria que vá por la singularidad cruza por primera vez el plano $\lambda=0$ ortogonalmente, entonces $\Gamma_{2}$ no pasa por $\xi_{1}$ (ni lo hace ninguna de las líneas pares) y es analítica [11].

\footnotetext{
${ }^{1}$ Utilizaré la misma notación para las familias $f$ que la de Markellos et. al. [30]
} 


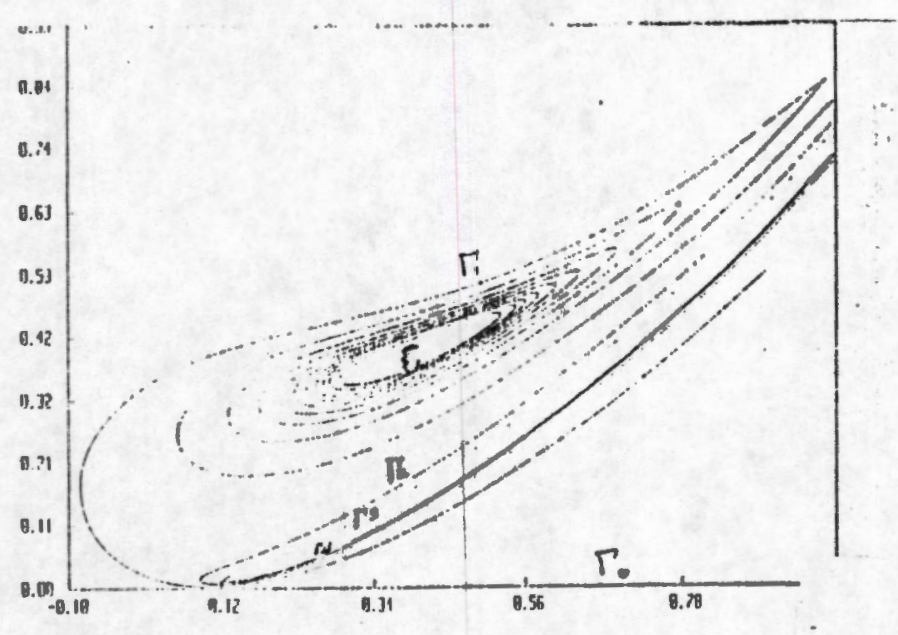

Figura 5.1: Lineas $\Gamma_{0}$ a $\Gamma_{6}$ para $\gamma_{1}=0.7857$

$\Gamma_{3}$ tiene también dos ramas espirales que convergen a un punto que denotaré por $\xi_{3}$ y que es la segunda intersección con el plano de Poincaré de la órbita que sale de la singularidad, es decir, $T \xi_{1}=\xi_{3}$. Las espirales alrededor de $\xi_{3}$ alcanzan a ser apreciables hasta la figura (5.6). De hecho, todas las líneas impares $\Gamma_{2 n+1}$ están formadas por dos ramas espirales que convergen a un punto $\xi_{2 n+1}$ y que es la n'esima intersección con el plano $\&$ de la órbita que sale de la singularidad.

En la figura (5.1) se muestran las líneas $\Gamma_{0}$ a $\Gamma_{6}$ para $\gamma_{1}=0.7857$ en el plano de Poincaré $\left(x_{1}, x_{2}=0, x_{3}, x_{4}=x_{4}\left(x_{1}, x_{2}, x_{3}\right)\right)$, con $x_{1}$ en el eje horizontal y $x_{3}$ en el eje vertical. Las líneas $\Gamma_{n}$ para $n=1, \ldots, 6$ son la reflexión respecto al eje $x_{1}$ de $\Gamma_{n}$ respectivamente. En todos los dibujos de líneas de simetría (exepto figura (5.2)) se utilizan los siguientes colores: $\Gamma_{1}, \Gamma_{-1}$ azul; $\Gamma_{2}, \Gamma_{-2}$ rojo; $\Gamma_{3}, \Gamma_{-3}$ verde; $\Gamma_{4}, \Gamma_{-4}$ naranja; $\Gamma_{5}, \Gamma_{-5}$ amarillo; $\Gamma_{6}, \Gamma_{-6}$ negro.

Como puede verse en la figura, las líneas $\Gamma_{2}, \Gamma_{3}, \ldots$, etc. se encuentran una dentro de otra y todas ellas dentro de los dos brazos salientes de $\Gamma_{1}$, de tal forma que no se produce ninguna intersección y no pueden existir órbitas periódicas.

Cuando $\gamma_{3}=\gamma_{1}{ }^{*}=0.788541$, todas las líneas se hacen tangentes en un solo punto parabólico.

Para valores de $\gamma_{1}$ que son un poco mayores que $\gamma_{1}{ }^{*}$ se producirá la intersección y nacerán (en parejas de puntos) infinitas órbitas periódicas. Particularmente, habrá dos órbitas de periodo 1 que forman una familia con la variación del parámetro $\gamma_{1}$ y que se le llama la familia principal $f_{0}$ [15] [28] 


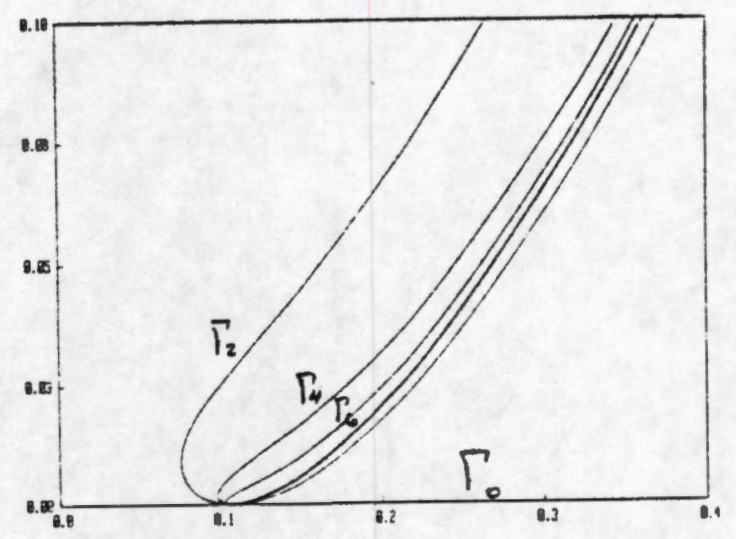

Figura 5.2: Líneas $\Gamma_{2}, \Gamma_{4}$ y $\Gamma_{6}$ para $\gamma_{1}=0.788541$

[30] [33] [42]. Estas dos órbitas nacen en sentidos opuestos y en la dirección tangente a todas las líneas de simetría (el eje $x_{1}$ ) en el punto de bifurcación.

Todas las líneas de simetría deben pasar por los dos puntos de período 1 de la familia principal (propiedad 8 cap. 4). El análisis de estabilidad muestra que la órbita de periodo 1 del lado izquierdo $f_{0}^{l}$ (el superíndice $l o$ $r$ indica que se trata de el punto sobre $\Gamma_{0}$ que tiene coordenada $x_{1}$ menor ó mayor respectivamente), es estable y la del lado derecho $f_{0}{ }^{r}$ es inestable.

En la figura (5.3) se muestran las líneas $\Gamma_{-6}, \ldots, \Gamma_{0}, \ldots, \Gamma_{6}$ para $\gamma_{1}=0.8$ $(C \cong 0.07629)$ y en la (5.4) una magnificación que incluye a las dos órbitas de la familia principal marcadas por $f_{0}^{l}$ y $f_{0}^{r}$, asi como órbitas de periodos 4,5 , 6,7 y 8 marcadas con un circulo pequeño. Las correspondientes trayectorias se encuentran en la figura (5.5).

Cabe hacer notar que la pareja de puntos de período par, por ejemplo $4^{l}$ y $4^{r}$ son de hecho la misma órbita (simétrica de período par con 2 puntos sobre $\Gamma_{0}$ ), mientras que la pareja de puntos de periodo impar por ejemplo $5^{l}$ y $5^{r}$ son necesariamente órbitas diferentes, por la propiedad 7 cap. 4 .

Cerca del punto elíptico de la famila principal $f_{0}^{l}$, el ángulo de rotación promedio $A$ (ver capitulo 4) da el ángulo promedio entre las líneas pares $\ldots \Gamma_{4}, \Gamma_{-2}, \Gamma_{0}, \Gamma_{2}, \Gamma_{4}$ o las impares ... $\Gamma_{-3}, \Gamma_{-1}, \Gamma_{1}, \Gamma_{3}, \ldots$ Este ángulo está relacionado con la traza del mapeo linearizado $L$ alrededor de la órbita estable $f_{0}$ :

$$
\operatorname{tr} L=2 \cos A
$$

Además, al igual que en el mapa estándárd [36], las líneas de simetria rotan 


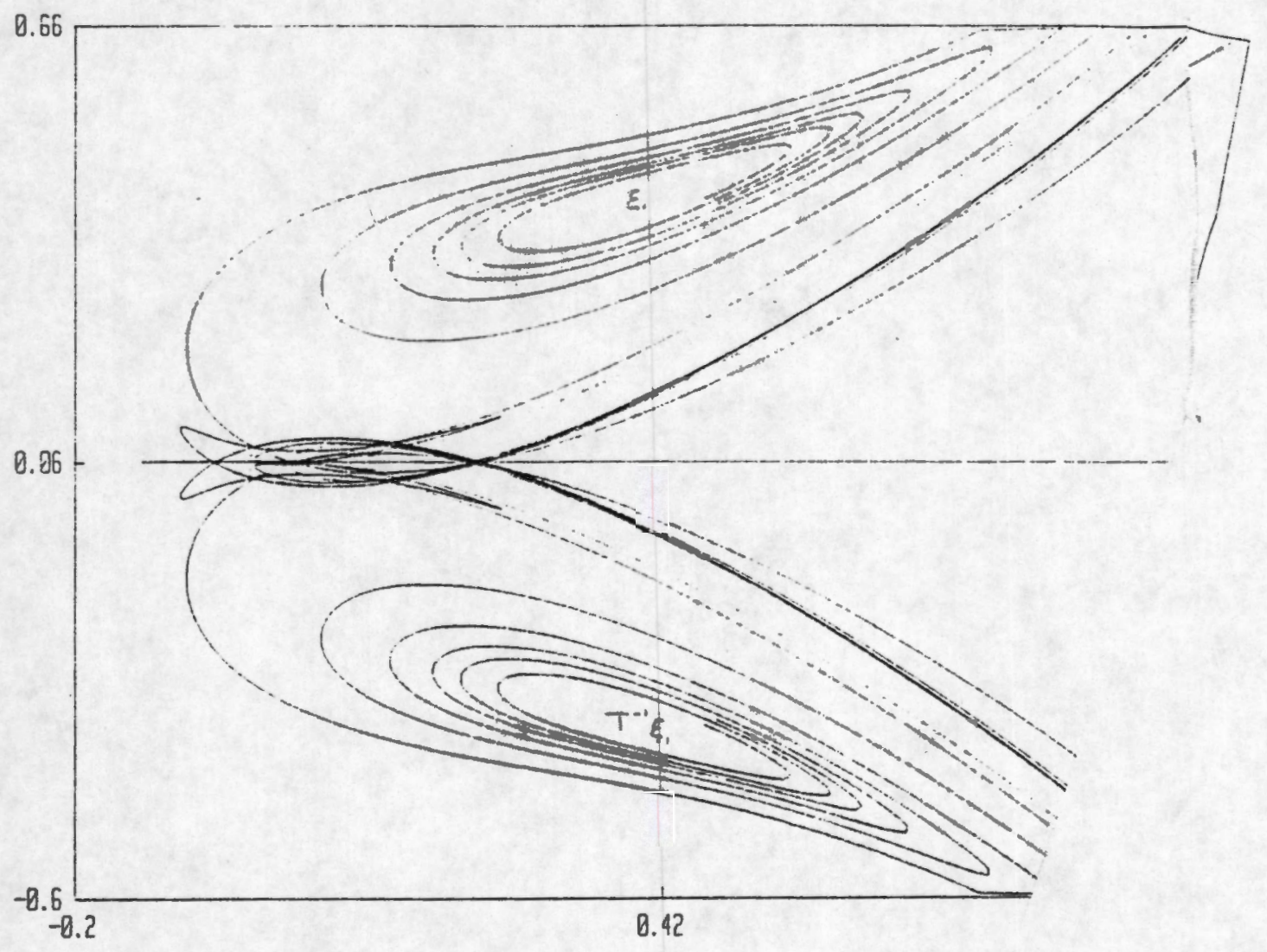

Figura 5.3: Líneas $\Gamma_{-6}, \ldots, \Gamma_{0}, \ldots, \Gamma_{6}$ para $\gamma_{1}=0.8$. 


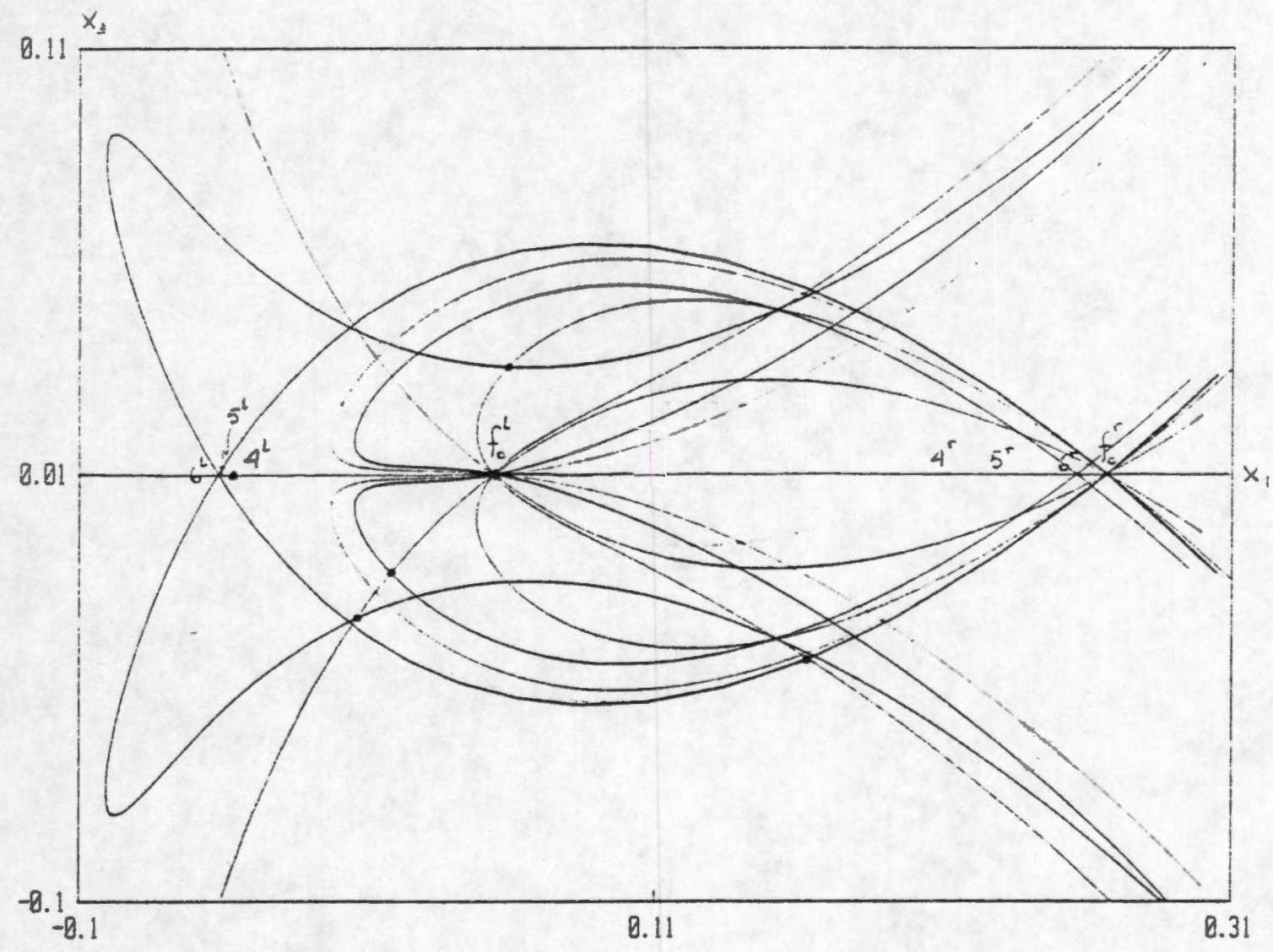

Figura 5.4: Magnificación de la figura anterior que incluye a las dos órbitas de la familia principal $f_{0}^{l}$ y $f_{0}^{r}$. Órbitas de periodo 4,5 y 6 se encuentran marcadas. 

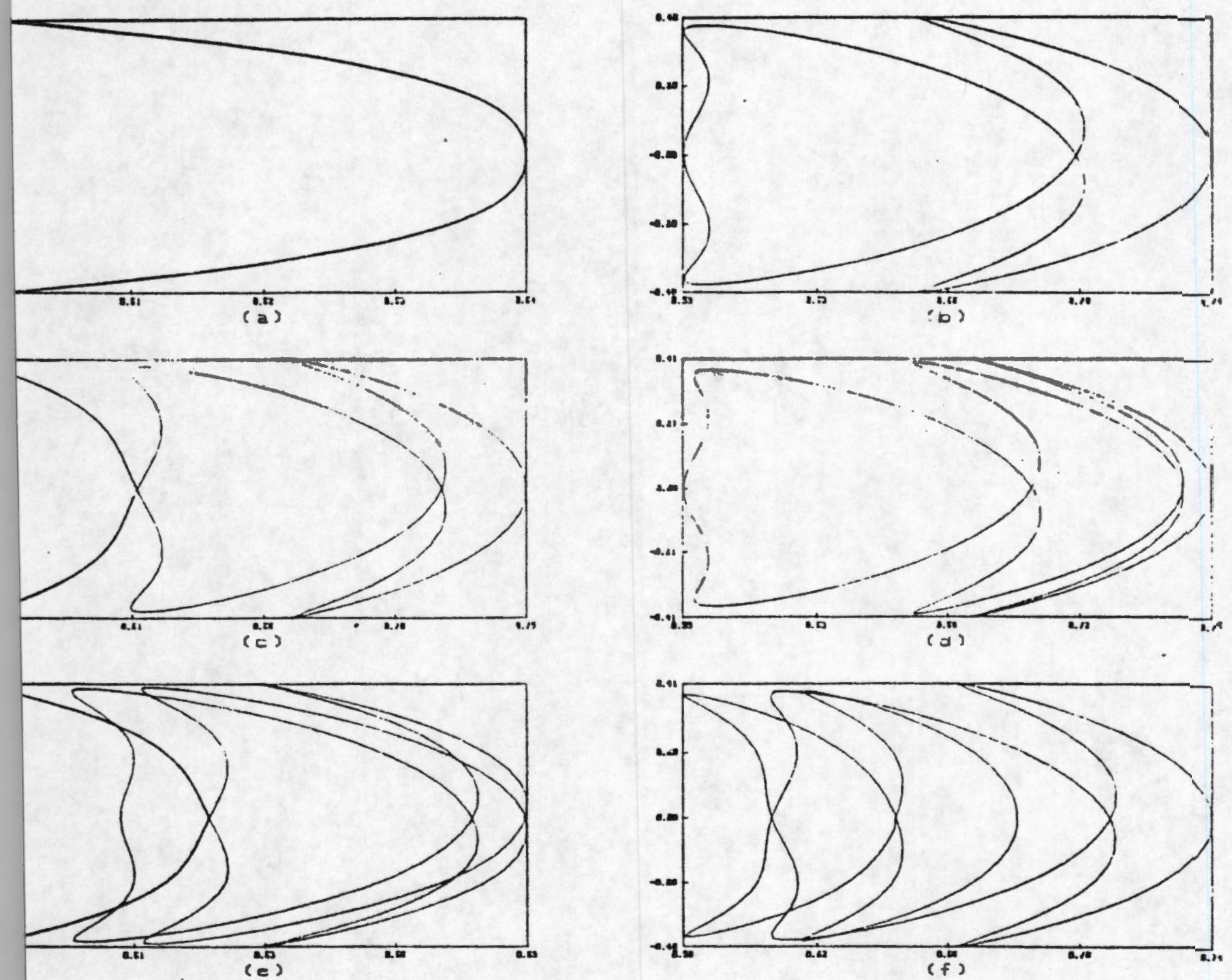

Figura 5.5: Órbitas periódicas en el plano meridiano para $\gamma_{1}=0.8$ con períodos $1,4,5,6,7$ y 8 en el espacio de configuración usando coordenadas cilindricas $\rho$ (eje horizontal) y $z$ (eje vertical). 
alrededor de $f_{0}^{l}$ en el sentido de las manecillas del reloj cuando $\gamma_{1}$ aumenta: las de subíndice mayor giran más rápido y rebasan a las de subindice más bajo. Se generan asi órbitas periódicas que nacen de $f_{0}^{\prime}$ con número de rotación $p / q$ (llamado el residuo en [18]):

$$
R=\frac{1}{4}(2-\operatorname{tr} L)=\sin ^{2} A / 2
$$

En el momento que dos órbitas de periodo $q$ están bifurcando de $f_{0}{ }^{\prime}$, las líneas $\Gamma_{q}$ y $\Gamma_{0}$ son tangentes de inflexión en el punto $f_{0}$.

Si el parámetro aumenta ligeramente, se producirán dos puntos nuevos de intersección entre $\Gamma_{q}$ y $\Gamma_{0}$, que son las dos órbitas de período $q$ que acaban de bifurcar de $f_{0}$. Esas órbitas permanecerán sobre $\Gamma_{0}$ al cambiar el parámetro $\gamma_{1}$ y forman una familia. Algunas familias de órbitas periódicas múltiples han sido estudiadas por Markellos et. al. [31] en un pequeño intervalo de valores de $\gamma_{1}$.

De esta manera, cuando $\gamma_{1}$ y $A$ crecen en forma continua, se obtendrán nuevos racionales $p / q$ dando infinitas órbitas periódicas, todas ellas nacidas de $f_{0}{ }^{\prime}$ por resonancia.

En la figura (5.4), la intersección del lado izquierdo de $\Gamma_{3}$ con $\Gamma_{0}$ es casj tangente, lo cual indica que un par de órbitas de periodo 3 está próximo a bifurcar de la órbita elíptica de la familia principal. Por otro lado, $\Gamma_{4}, \Gamma_{5}$ y $\Gamma_{6}$ intersectan en cuatro puntos a $\Gamma_{0}$. Dos de ellos son las órbitas de la familia principal (por donde pasan todas las líneas de simetría), y los otros dos son órbitas de periodos 4,5 y 6 respectivamente, que bifurcaron de $f_{0}^{l}$. Las órbitas periódicas de períodos 2 y 3 aún no han nacido para este valor del parámetro $\left(\gamma_{1}=0.8\right)$.

Si se continúa aumentando $\gamma_{1}$, la intersección (del lado izquierdo) entre $\Gamma_{2}$ y $\Gamma_{0}$ se vuelve una tangencia de inflexión, y la correspondiente órbita de la familia principal se desestabiliza. Dos puntos de periodo 2 nacen en la dirección de la tangencia (el eje $x_{1}$ ), y determinan la llamada familia $f_{1}$ (que bifurca de la familia principal). Así, la última órbita que nace de $f_{0}{ }^{l}$ es la de periodo 2. Para $\gamma_{1}$ un poco mayor aún, la órbita $f_{0}{ }^{\prime}$ será hjperbólica, y habrán cuatro puntos de intersección de $\Gamma_{0}$ con $\Gamma_{2}$ : dos de ellos inestables y pertenecientes a $f_{0}$, con período 1 , y dos de período 2 pertenecientes a la familia $f_{1}$.

En la figura (5.6) se muestran las líneas $\Gamma_{j}$ con $j$ de -6 a 6 cuando $\gamma_{1}=0.85$. La órbita $f_{1}$ ya está presente y $f_{0}{ }^{l}$ es inestable. En la figura (5.7) 


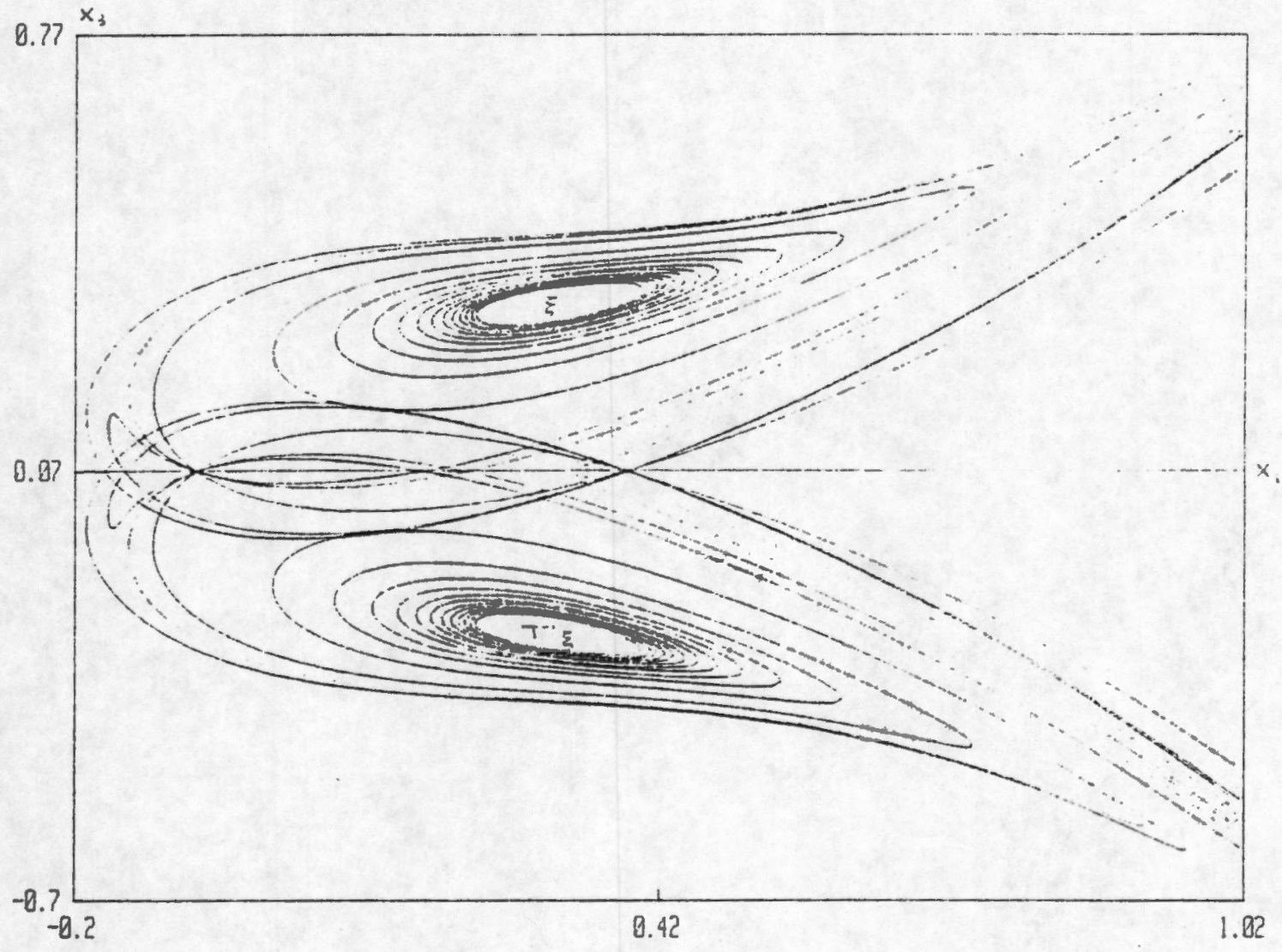

Figura 5.6: Lineas $\Gamma_{-6}$ a $\Gamma_{6}$ para $\gamma_{1}=0.85$. 


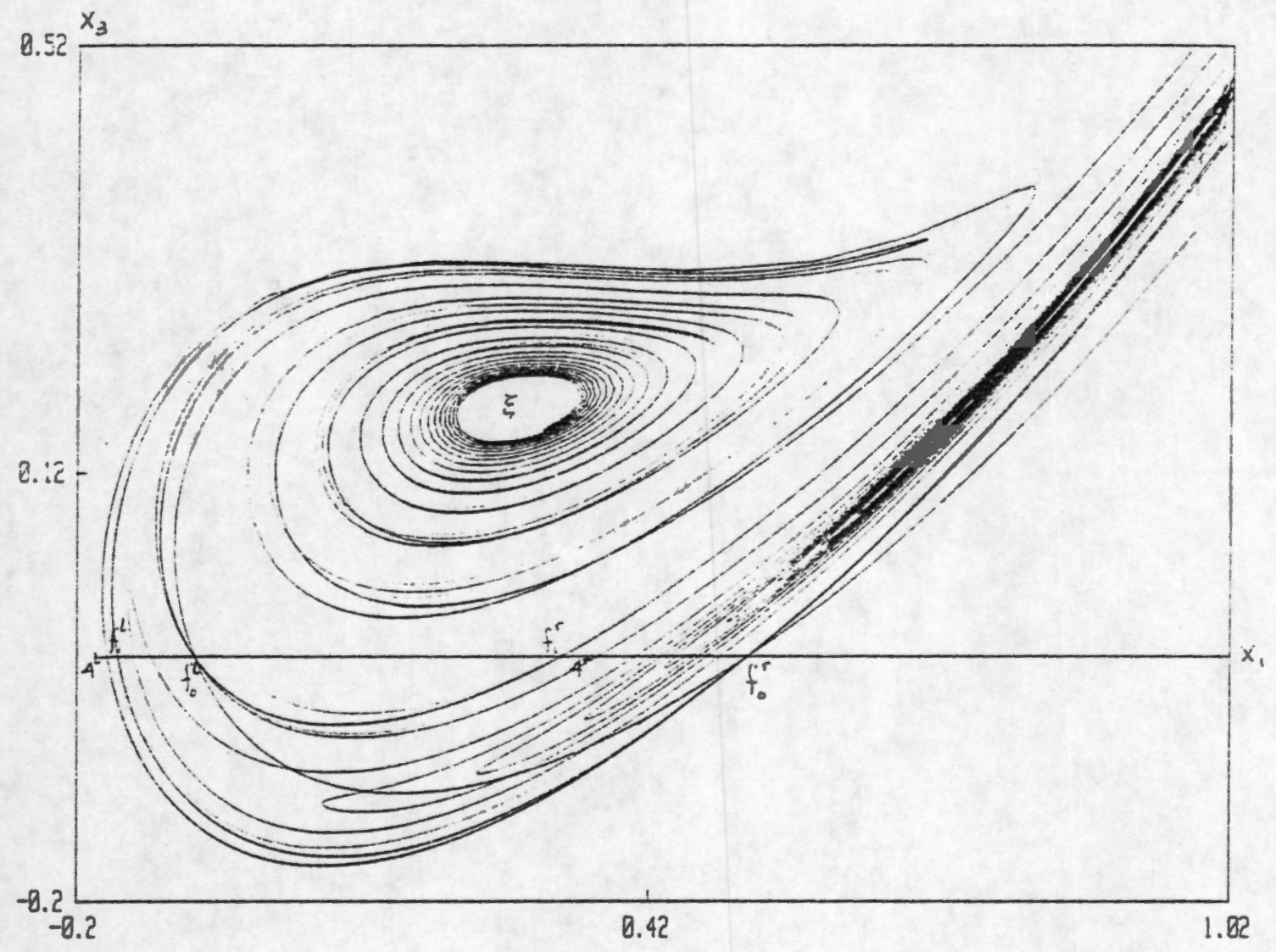

Figura 5.7: Lineas $\Gamma_{0}$ a $\Gamma_{4}$ para $\gamma_{1}=0.9$ 

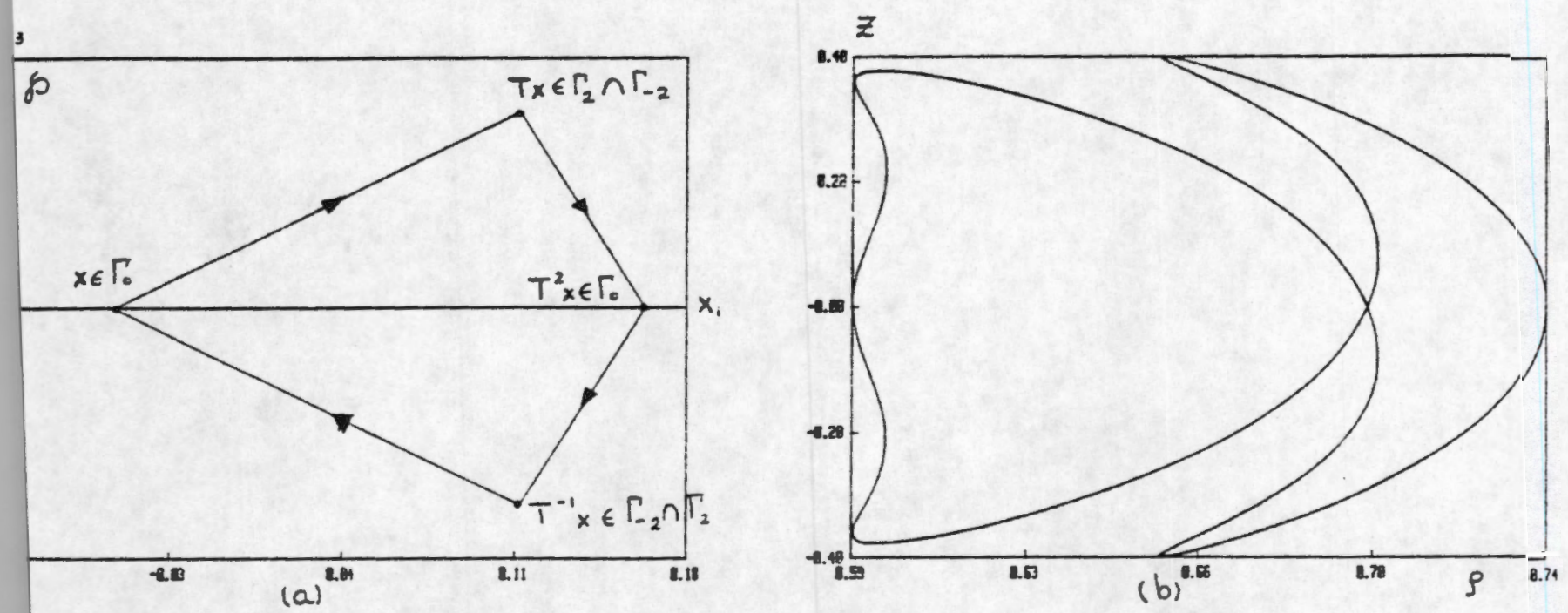

Figura 5.8: a) Órbita de período 4 en el plano $p$ con dos puntos $\mathrm{x}$ y $T^{2} \mathrm{X}$ sobre $\Gamma_{0}$. b) La trayectoria correspondiente en el plano meridiano $M$ es simétrica y cerrada.

sólo se muestran las líneas $\Gamma_{0}$ a $\Gamma_{4}$ para $\gamma_{1}=0.9$.

De acuerdo a la propiedad 9 del capítulo 4 , la línea $\Gamma_{2}$ se intersecta con $\Gamma_{1}$ sólo en puntos sobre $\Gamma_{0}$, como puede verificarse en las figuras. La propiedad 7 se ejemplifica en las figuras (5.8) a (5.10) para $\gamma_{1}=0.8$, con sus respectivas trayectorias en el espacio de configuración. En la figura (5.8) se muestra una órbita de periodo par (4) con dos puntos sobre $\Gamma_{0}$. La trayectoria en el espacio de configuración $\mathbf{M}$ es simétrica cerrada. La figura (5.9) muestra una órbita de período par (4) con dos puntos sobre $\Gamma_{1}$. Esta órbita es asimétrica abierta en M. Finalmente, en la figura (5.10) se muestran una órbita de período impar (5) con un punto sobre $\Gamma_{0}$ y otro sobre $\Gamma_{1}$. Estas son órbitas simétricas abiertas en $\mathbf{M}$. 
$x_{3}$

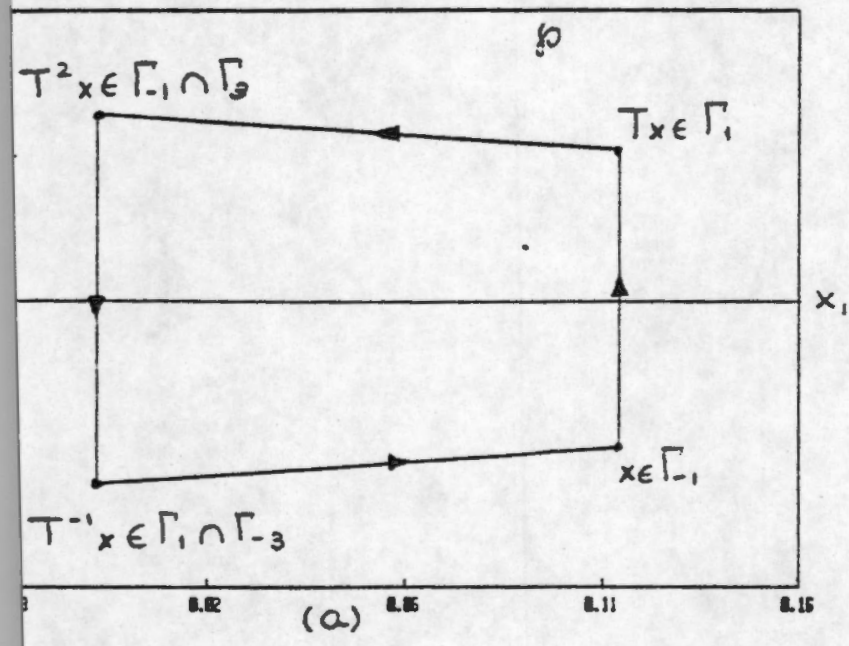

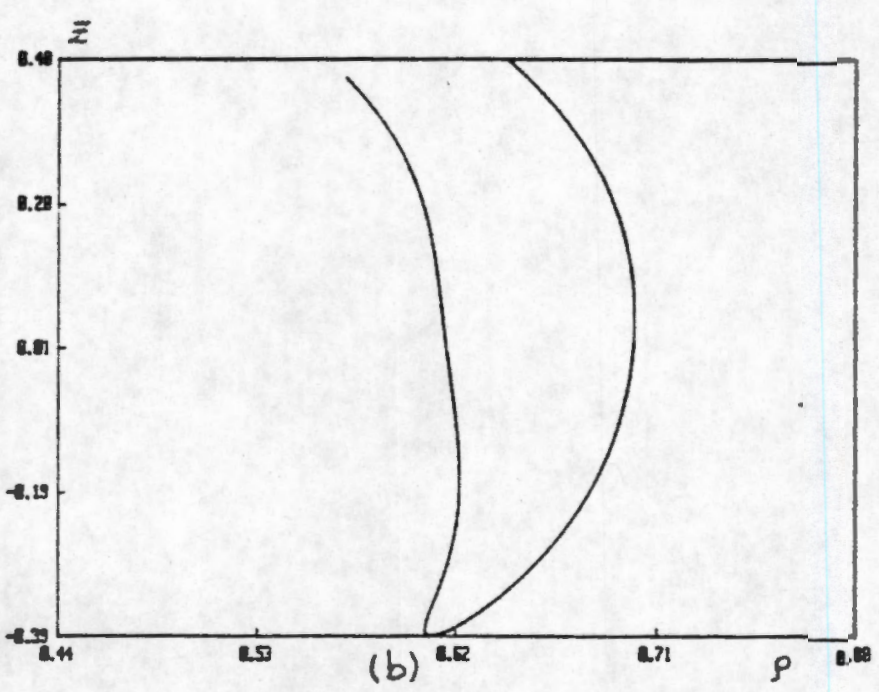

Figura 5.9: a) Órbita de período 4 en el plano $\wp$ con dos puntos $x$ y $T^{2} x$ sobre $\Gamma_{-1}$ (y dos puntos $T \times$ y $T^{-1} \times$ en $\Gamma_{1}$ ). b) La trayectoria correspondiente en $\mathbf{M}$ es asimétrica y abjerta.
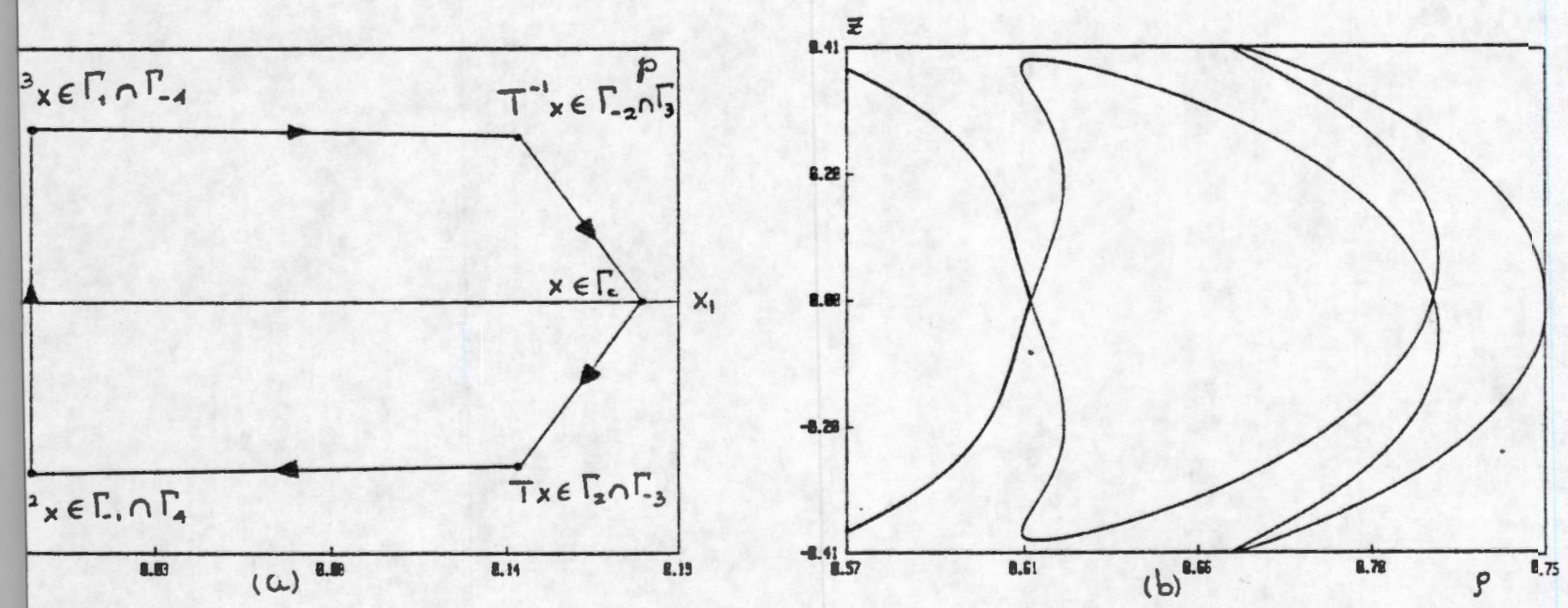

Figura 5.10: a) Órbita de periodo 5 en el plano $\wp$ con un punto $\mathrm{x}$ sobre $\Gamma_{0}$ y otro $T^{3} \mathrm{x}$ sobre $\Gamma_{1}$. b) La trayectoria en $M$ es simétrica y abierta. 


\section{2 Órbitas simétricas simples}

En esta sección y la siguiente se enfoca el estudio a las líneas $\Gamma_{1}$ y $\Gamma_{2}$ al aumentar el parámetro para estudiar la estructura global de las órbitas periódicas simples.

Introduzco el espacio de parámetros (que denotaré por $\Sigma$ ) utilizado en Markellos et. al. [30] para hacer una correspondencia directa con ese estudio, donde el parámetro que se usa es $C=(1 / 2)\left(1 /\left(2 \gamma_{1}\right)^{4}\right)$ (la energía). Para cada valor de $C, y$ por consiguiente de $\gamma_{1}$, se grafica la coordenada radial $2 \gamma_{1} u$ de las órbitas periódicas en el punto donde cruzan perpendicularmente al ecuador (ie. $u^{\prime}=0$ ó $x_{3}=0$ ). Se obtiene así una gráfica global de las familias de órbitas periódicas en el espacio de parámetros $\Sigma$.

Para analizar la dinámica de las líneas $\Gamma_{1}$ y $\Gamma_{2}$ asi como de sus intersecciones, introduzco la siguiente notación: sea $S_{1}$ la vuelta más externa de $\Gamma_{1}$, y sea $S_{n}$ la $n$ 'ésima vuelta de $\Gamma_{1}$. Análogamente, sea $S_{-n}$ la n'ésima vuelta de $\Gamma_{-1}$ (ver figuras $(5.11)$ y (5.12)).

El mecanismo por el cual nace cualquier familia $f_{2 n}(n \in Z)$ de período 1 es esencialmente el mismo que el de la familia principal $f_{0}$ pero se presentará. nuevamente para mayor claridad.

Conforme el parámetro aumenta, el punto $\xi_{1}$ baja hacia $\Gamma_{0}$, produciéndose un número creciente de intersecciones entre $\Gamma_{1}$ y $\Gamma_{0}$. Cuando $S_{n}$ (y también $S_{-n}$ ) es tangente a $\Gamma_{0}$, nace la familia $f_{2(n-1)}$ de órbitas de período 1 . Como en el caso de la familia principal, cuando una nueva familia $f_{2(n-1)}$ nace, todas las líneas de simetría son tangentes en el mismo punto, el punto de bifurcación. Si el parámetro aumenta ligeramente, $\Gamma_{1}$ y $\Gamma_{0}$ se intersectan en dos puntos (de la familia $f_{2(n-1)}$ ): uno elíptico (del lado izquierdo sobre el eje $x_{1}$ ) que denotaré por $f_{2(n-1)}$ y otro hiperbólico (del lado derecho) que denotaré por $f_{2(n-1)}{ }^{r}$. Todas las líneas de simetria deben pasar por estos dos puntos. Cerca del punto $f_{2(n-1)}{ }^{l}$ cualquier línea $\Gamma_{i}$ rota en el sentido de las manecillas del reloj cuando $\gamma_{3}$ aumenta. Eventualmente, ésta se hace tangente de inflexión con $\Gamma_{0}$, intersectándose después en dos puntos de periodo $i$ que bifurcan de $f_{2(n-1)}$. Es asi como el punto elíptico de la familia $f_{2(n-1)}$ bombea órbitas periódicas múltiples. La última que nace es la de periodo 2 , cuando $\Gamma_{2}$ se hace tangente a $\Gamma_{0}$, que se conoce como la familia $f_{2 n-1}$ de trayectorias cerradas [30].

En la figura (5.13) se ilustra la dinámica de las lineas de simetría en 


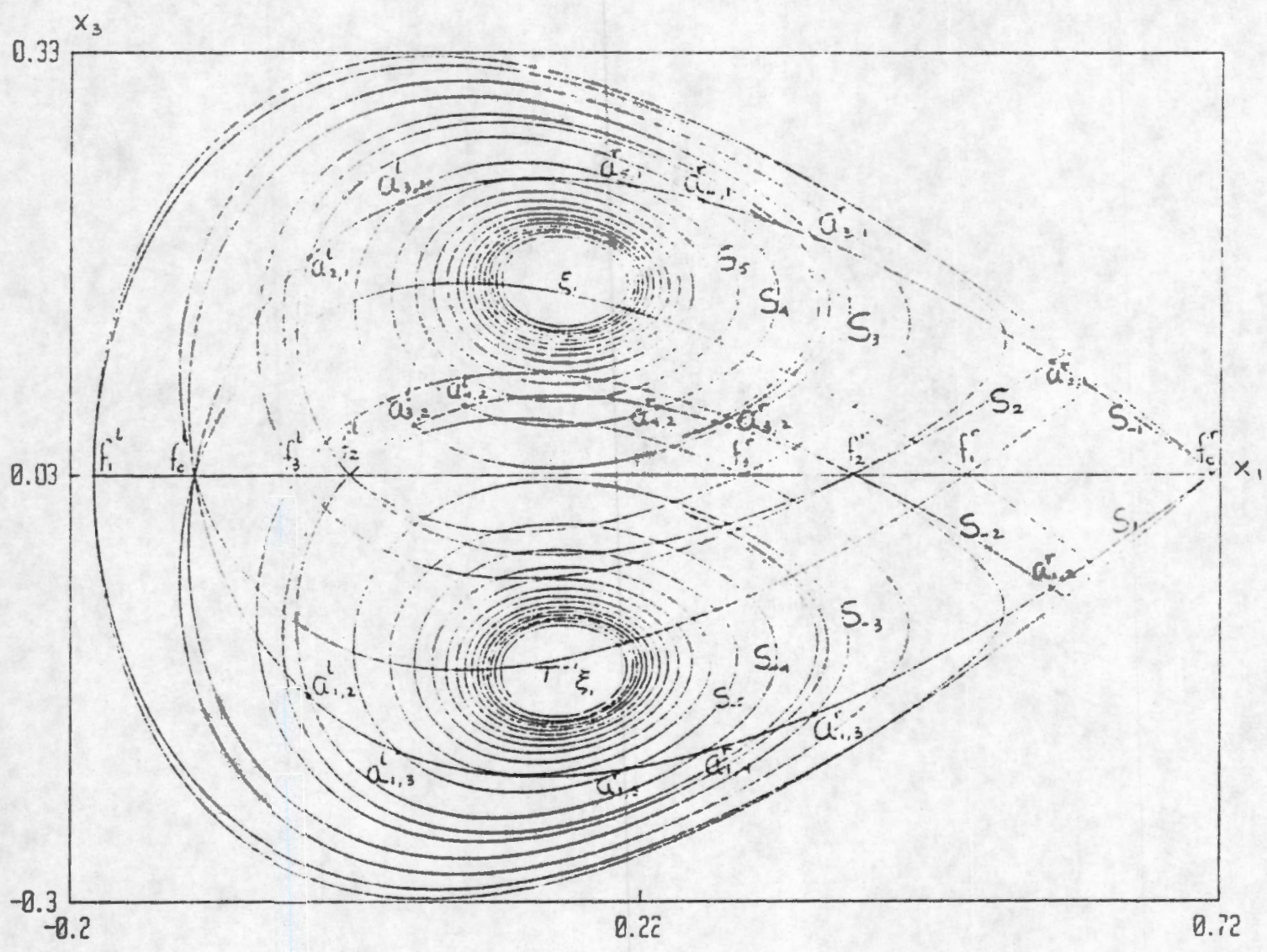

Figura 5.11: Lineas $\Gamma_{j}$ para $j$ de -2 a 2 cuando $\gamma_{1}=1.0$ 


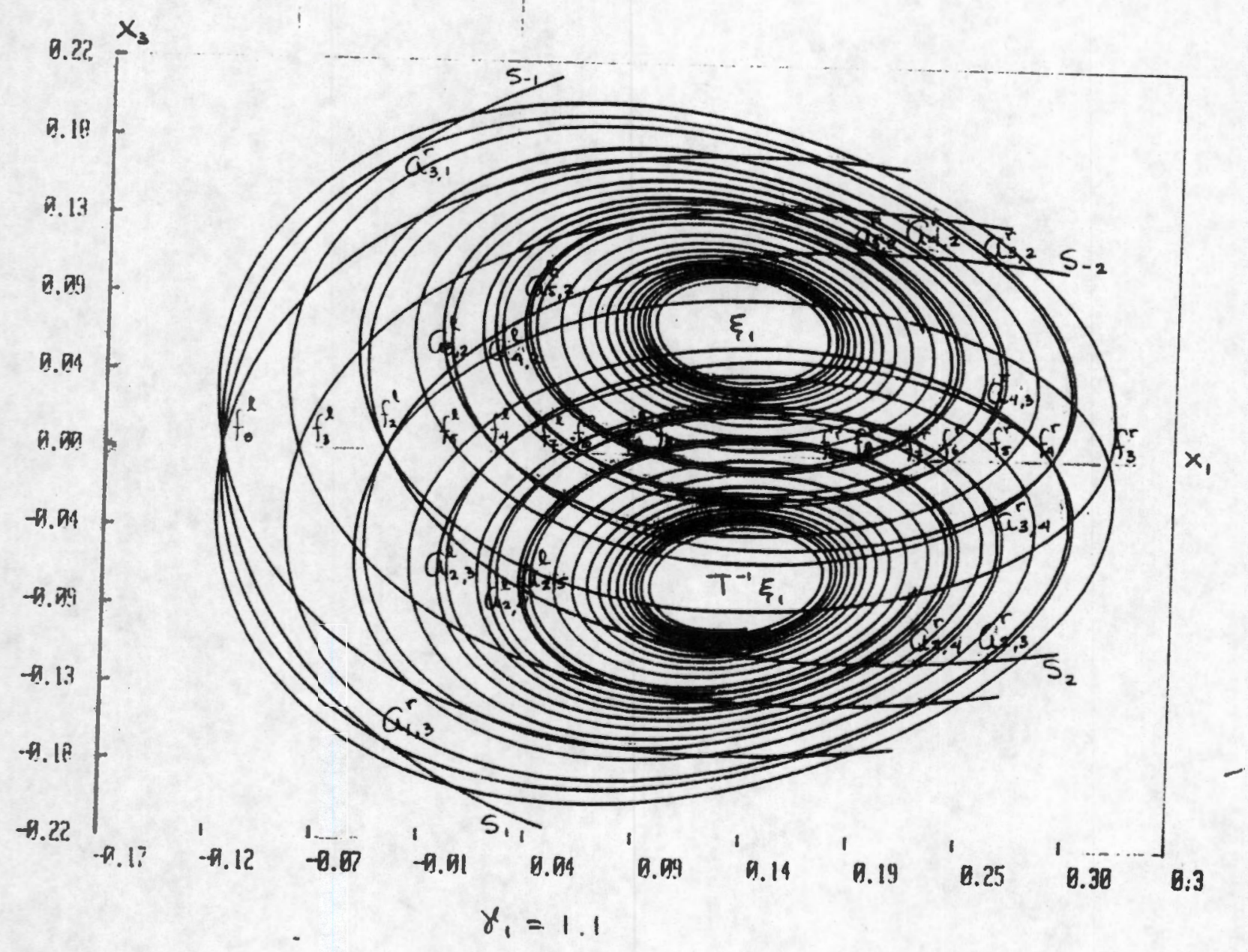

Figura 5.12: Líneas $\Gamma_{j}$ para $j$ de -2 a 2 cuando $\gamma_{1}=1.1$ 

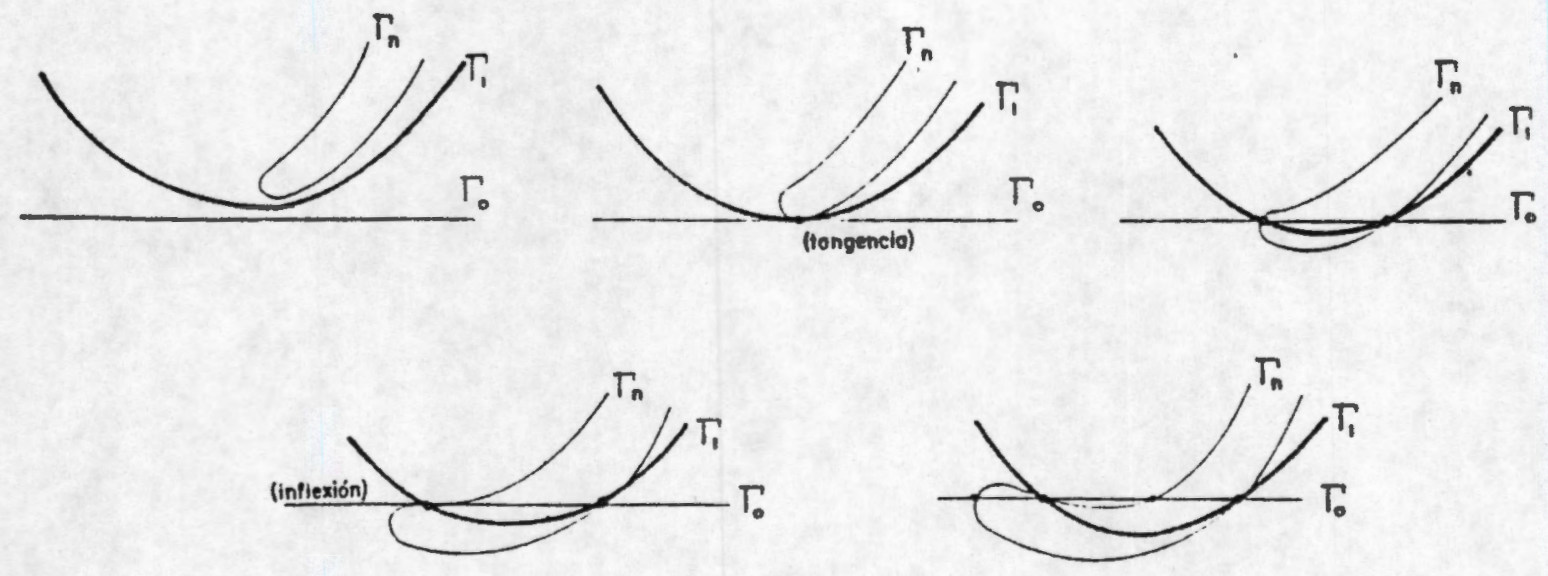

Figura 5.13: Secuencia típica de bifurcación de dos puntos de período $i$ alrededor del período 1

una secuencia típica de bifurcaciones que da lugar al nacimiento de un par de período 1 y de cualquier periodo $i \in N$ alrededor del período 1 estable cuando $\gamma_{1}$ aumenta.

Por otro lado, se observa en la figura (5.11) que cuando $\gamma_{1}=1$ la órbita $f_{0}{ }^{\top}$ alcanza el punto crítico $x=\log 2, \lambda=0$ y se vuelve una órbita ecuatorial (la órbita circular, sección 2.1). Vallarta [42] le llamó la órbita periódica más externa. Todas las líneas de simetría pasan por el punto crítico cuando $\gamma_{1}=1$.

Para valores de $\gamma_{1}>1$, la región de Hill consta de dos zonas disconexas: una infinita y otra finita. En la región infinita no pueden existir órbitas periódicas [15], [30], por lo que ya no nos ocuparemos de ella. La región finita se hace más pequeña conforme $\gamma_{1}$ aumenta, a la vez que el punto $\xi_{1}$ se acerca a $\Gamma_{0}$ generándose nuevas familias $f_{2 m}$, con $n$ un número creciente, así como infinidad de órbitas periódicas múltiples que bifurcan de su parte estable. Por otro lado, conforme $\gamma_{1}$ aumenta desde su valor 1 , las líneas parecen "desenredarse": el punto de intersección de $\Gamma_{n}$ con $E$ (la frontera de la región permitida en el plano de Poincaré) se desplaza en el sentido de las manecillas del reloj a lo largo de $E$ si $n>0$ (y en sentido contrario si $n<0)$, y las órbitas periódicas externas empezarán a desaparecer del plano $\wp$ para convertirse en órbitas ecuatoriales. Esto ocurre cuando el punto de 


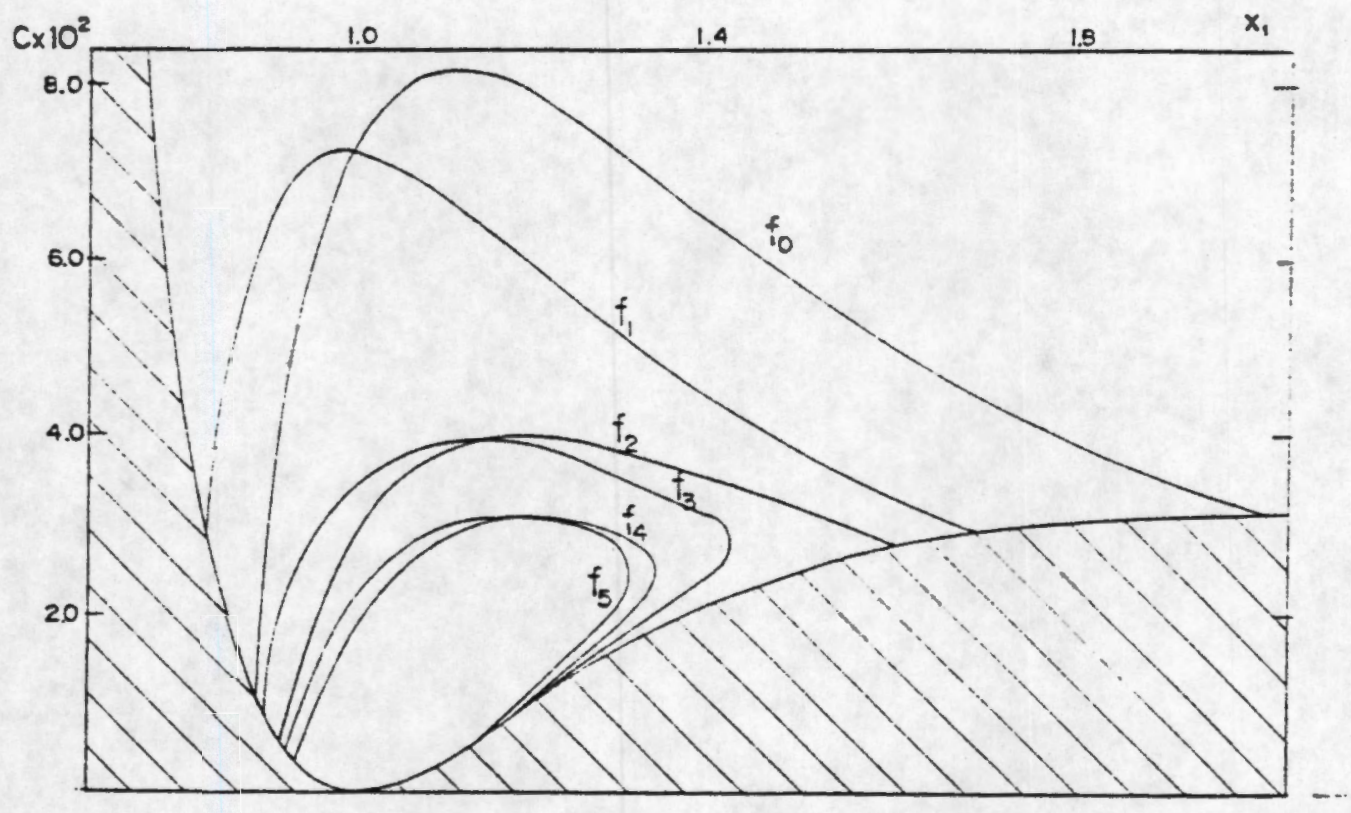

Figura 5.14: Familias $f_{0}, f_{1}, \ldots, f_{5}$ obtenidas por Markellos et. al.. El eje horizontal es $2 \gamma_{1} u$, y el eje vertical $C=\left(1 /\left(2 \gamma_{1}\right)^{4}\right) / 2$. El máximo de una familia $f_{2 i}$ corresponde a un punto en que todas las líneas de simetría son tangentes.

intersección de $\Gamma_{n}$ con $\Gamma_{0}$ queda en la frontera de la región permitida $E$. Esto tiene conexión con el estudio de la ecuación de Hill para obtener órbitas infinitamente vecinas al ecuador hecho por De Vogelaere [10].

La no intersección de $\Gamma_{1}$ con $\Gamma_{2}$ excepto en puntos sobre $\Gamma_{0}$, aunado al hecho de que una línea de simetría no se intersecta a sí misma, dan la base para calcular el orden de desaparición de las familias de órbitas simétricas simples en órbitas ecuatoriales cuando $\gamma_{1}$ aumenta: $f_{0}{ }^{r},\left(f_{1}{ }^{l}, f_{1}{ }^{r}\right),\left(f_{2}{ }^{r}, f_{0}{ }^{l}\right)$, $\ldots,\left(f_{n}{ }^{l}, f_{n}{ }^{r}\right),\left(f_{n+1}{ }^{r}, f_{n-1}{ }^{l}\right)$, etc., ( $n$ impar $)$, donde $($,$) indica que este criterio$ no es suficiente para decidir cuál de ellas desaparece primero. En realidad, $f_{n}{ }^{l}$ y $f_{n}{ }^{r}$ son la misma órbita, y ambas desaparecen en el mismo valor de $\gamma_{1}$. Por otro lado, Markellos et. al. [28] mostraron que $f_{n+1}{ }^{r}$ y $f_{n-1}{ }^{\prime}$ ( $n$ impar) se vuelven ecuatoriales en el mismo valor de $\gamma_{1}$ aún cuando ellas pertenecen a diferentes familias.

Cuando $\gamma_{1} \gg 1$, la región finita en el plano de Poincaré tiende a $x_{1}=$ $x_{3}=0$, y las órbitas en el espacio de configuración se mueven aproximándose 
cerca del dipolo. 


\section{3 Órbitas asimétricas simples}

Las órbitas de período par del tipo abjerto con dos puntos sobre $\Gamma_{3}$ son órbitas asimétricas en el espacio de configuración si bien son simétricas bajo $I_{0}$ en el plano de Poincaré $\rho$ (ver figura (5.9)).

Al igual que las órbitas periódicas simétricas, las asimétricas pueden ser simples ó múltiples, según si su período es 2 ó un número par mayor que 2 respectivamente. Todas las intersecciones de las líneas impares que no estén sobre $\Gamma_{0}$ serán órbitas asimétricas.

Las órbitas asimétricas simples se obtienen de la intersección de $\Gamma_{1}$ con $\Gamma_{-1}$. La primera órbita nace cuando $S_{1}$ hace tangencia con $S_{-2}$, y conforme el parámetro crece, se producirán nuevas intersecciones entre $S_{1}$ y $S_{-n}$.

Sea $a_{m, n}$ ( $m$ y $n$ enteros positivos) el par de órbitas que nace de la intersección de $S_{m}$ con $S_{-n}$. Nótese que si $m<n$ las intersecciones estarán en el

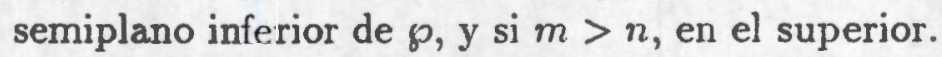

La órbita $a_{n, m}$ es simétrica a $a_{m, n}$ en el plano $\wp$ respecto a $I_{0}$, puesto que $a_{n, m} \in \Gamma_{1} \cap \Gamma_{-1}$. Puesto que $x_{4}>0$ en las condiciones iniciales, $T a_{n, m}=$ $a_{m, n}$ tendrá $x_{4}<0$. Por otro lado, si $a_{m, n}\left(x_{4}>0\right)$ es el punto inicial, su correspondiente órbita en el plano $(\rho, z)$ es la reflexión con respecto al eje horizontal (el ecuador) de la órbita con punto inicial $a_{n, m}\left(x_{4}>0\right)$, y no son la misma órbita puesto que son asimétricas.

Cuando $S_{1}$ cruza $I_{0} \xi_{1}\left(=T^{-1} \xi_{1}\right)$, habrán nacido infinitos pares de órbitas asimétricas, $y$ a partir de ese momento (conforme $\gamma_{1}$ aumente) comenzarán a desaparecer también en pares, debido a que $S_{1}$ se mueve hacia abajo de $\xi_{1}$, perdiéndose intersecciones que se habian generado anteriormente.

No obstante, un par de órbitas asimétricas simples que nacen juntás no desaparecen juntas, debido a la forma espiral de $\Gamma_{1}$ : si $a_{1, n}{ }^{r}$ y $a_{1, n}{ }^{l}$ son las órbitas del lado derecho e izquierdo de $a_{1, n}$ respectivamente, entonces $a_{1, n}{ }^{l}$ y $a_{1, n}{ }^{r}$ nacen juntas (por definición), pero $a_{1, n}{ }^{l}$ desaparece con $a_{1, n+2}{ }^{r}$, mientras que $a_{1, n}{ }^{r}$ con $a_{1, n-2}{ }^{l}$. Entonces, estas órbitas asimétricas simples son miembros de dos familias: $a_{1,2 n}$ y $a_{1,2 n+1}$ (para $n$ cualquier entero positivo), correspondientes a las intersecciones de las dos espirales de $\Gamma_{1}$ con $S_{1}$.

Un análisis similar se puede hacer para las intersecciones de $S_{m}$ y $S_{-n}$ para $m$ fijo y $n$ cualquier entero positivo tal que $n>m$. Habrán dos familias: $a_{m, p e r}$ y $a_{m, i m p a r}$, donde par e impar son cualesquier enteros positivos pares e impares mayores que $m$ respectivamente. 
Markellos et. al. [29] encontraron órbitas periódicas asimétricas simples, y denotaron por $a\left(f_{2 n}\right)$ a la familia de órbitas asimétricas que bifurcan de la familia $f_{2 n}(n \geq 0)$, ie., aquellas que son las intersecciones de $S_{n+1}$ con $\Gamma_{-1}$ (o de $S_{-(n+1)}$ con $\left.\Gamma_{1}\right)$ y que denoto por $a_{n+1, \text { par }} a_{n+1, \text { impar. }}$ Particularmente, en ese artículo estudiaron la familia asimétrica principal $a\left(f_{0}\right)$, que corresponde a las familias $a_{1, \text { par }}$ y $a_{1, \text { impar }}$, aunque no distinguen entre ellas. Ellos muestran gráficamente algunas órbitas asimétricas que pertenecen a la familia asimétrica principal, y una figura de la curva característica de esta familia para afirmar que las órbitas dibujadas pertenecen a la misma familia. Sin embargo, también anticiparon que las órbitas podrían no ser de la misma familia. Por otro lado, también encontraron órbitas asimétricas simples cerradas, que no se pueden obtener de intersecciones de las líneas de simetría puesto que no son simétricas respecto a $I_{0}$ en el plano de Poincaré, aunque podrían ser simétricas respecto a otra factorización del mapeo $T$ en dos involuciones. De hecho, puesto que existen otras simetrias en el problema de Störmer, éste podría ser el caso.

Obtuve también órbitas asimétricas múltiples (de periodo par mayor que dos) intersectando l' ineas $\Gamma_{n}$ y $\Gamma_{m}$ con $n$ y $m$ enteros impares, $|n-m|>2$. De hecho, hay una infinidad de familias asimétricas múltiples, que pueden clasificarse en base a las líneas de simetría. Su estudio y clasificación se hará posteriormente. En la figura (5.16) se muestran dos órbitas asimétricas múltiples con períodos 4 y 6 . 

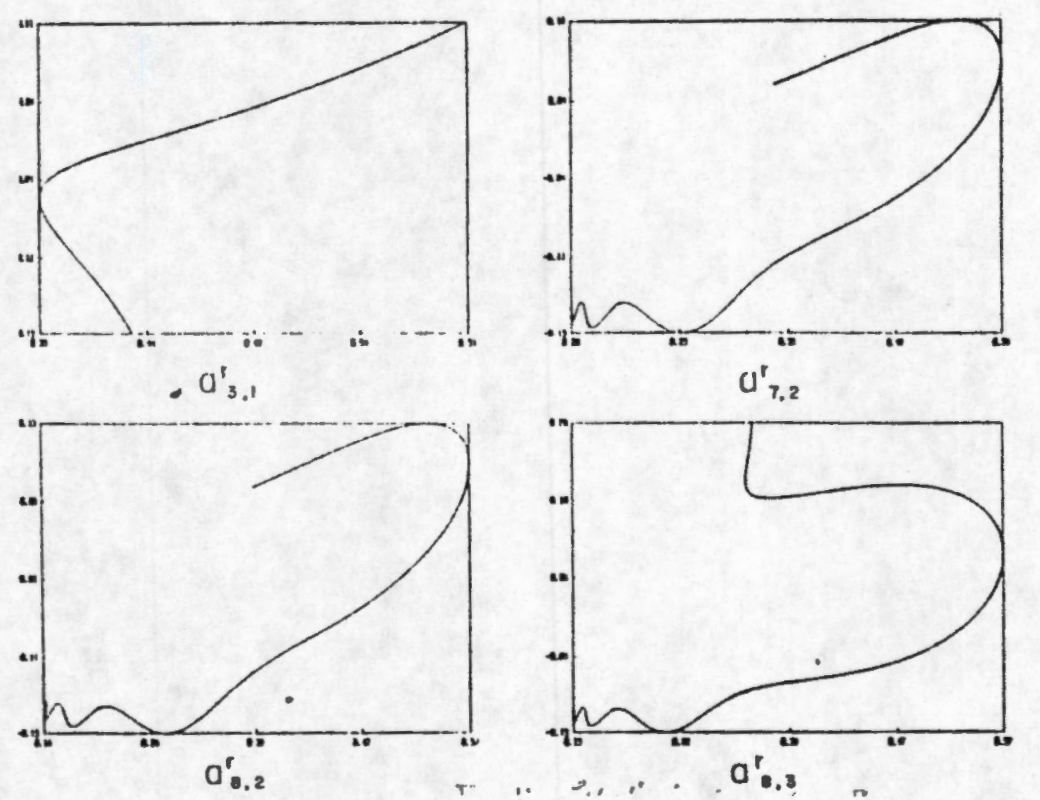

Figura 5.15: Órbitas asimétricas simples para $\gamma_{1}=1.1$
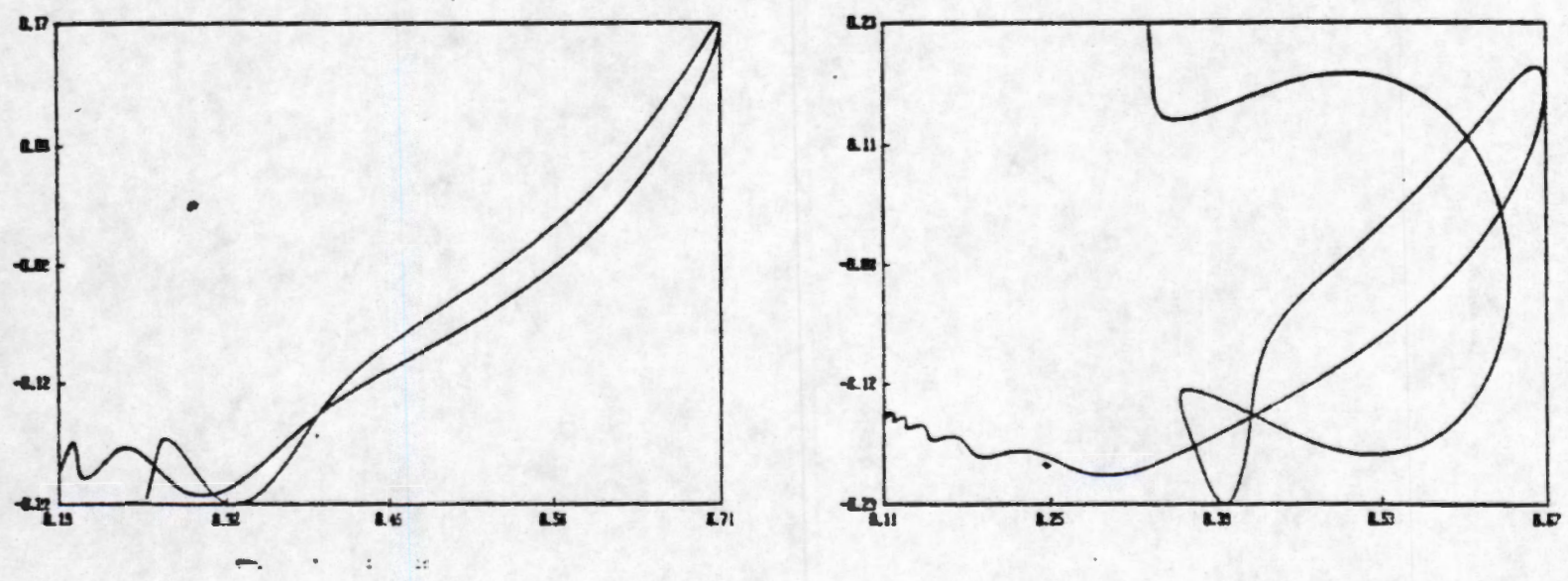

Figura 5.16: Órbitas asimétricas múltiples de periodos 4 y 6 


\subsection{Forma de las trayectorias periódicas sim- ples}

Graef [17] estudió los tipos de extremos de una variable con respecto a otra que pueden haber en el espacio de configuración (el plano meridiano): aplicó el principio de Maupertuis y encontró una ecuación para la trayectoria en el plano $(\rho, z)$. Después encontró tres curvas donde la primera y segunda derivadas de $\rho$ con respecto a $z$ y de $z$ con respecto a $\rho$ se anulan. Estas curvas dividen el plano en ocho regiones donde se encuentra un extremo de una variable respecto de la otra. Dos de estas curvas son el ecuador y el thalweg. La tercera está dada por:

$$
2 \gamma_{1} u=\cos ^{2} \lambda\left(3 \cos ^{2} \lambda-1\right)
$$

o en coordenadas cilíndricas

$$
\frac{\rho^{2}}{u^{3}}\left(3 \frac{\rho^{2}}{u^{3}}-1\right)=2 \gamma_{1}
$$

donde $u^{2}=\rho^{2}+z^{2}$

Se obtuvieron las trayectorias de las órbitas periódicas simples en el plano meridiano $(\rho, z)$. Se observa que un miembro típico de la familia $f_{n+2}$ tiene una oscilación más alrededor del thalweg que un miembro típico de $f_{n}$. En la figura (5.17) se muestran cuatro órbitas periódicas simétricas, pertenecientes a $f_{4}, f_{5}, f_{6}$ y $f_{7}$. En general, una órbita de la familia $f_{2 n+1}$ tiene $2 n+1$ regiones interiores. Obsérvese por ejemplo la órbita $f_{7}$, que tiene 7 regiones interiores. Se observa que las formas de las trayectorias concuerdan con el estudio de Graef. En la figura (5.18) se muestran las 8 regiones de Graef $\left(\gamma_{1}=1.1\right)$ con el tipo de extremo que debe haber en cada una mostrado en forma descriptiva, y las órbitas simétricas de la figura (5.17) superpuestas.

Con las órbitas asimétricas ocurre algo similar: las intersecciones de $S_{m}$ y $S_{-n}$ para $m$ fijo y cualquier $n$, ambos enteros positivos tales que $m<$ $n$, producen órbitas periódicas en el plano $(\rho, z)$ con un número creciente de oscilaciones alrededor del thalweg en el semiplano superior, cuando $n$ aumenta.

Esto era de esperarse debido a que conforme $\gamma_{1}$ aumenta, la curva $S_{-n}$ que intersecta a $\Gamma_{0}$ (ó a $S_{m}$ ) tiene a $n$ cada vez mayor (puesto que $\xi_{1}$ se acerca a $\Gamma_{0}$ ), y las intersecciones que se producen están cada vez más cerca 

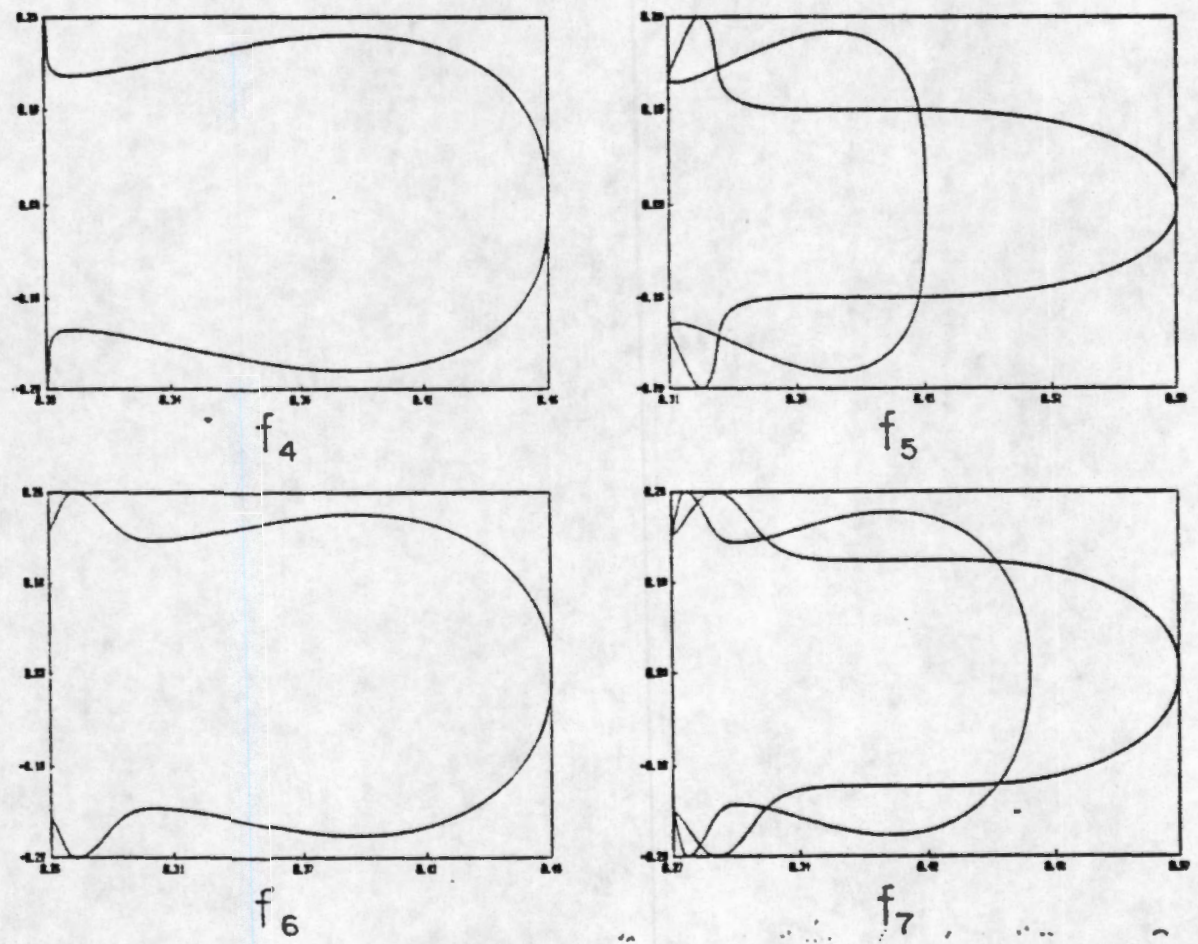

Figura 5.17: Trayectorias típicas de las familias $f_{4}, f_{5}, f_{6}, f_{7}$ en el plano meridiano $\left(\gamma_{1}=1.1\right)$. 


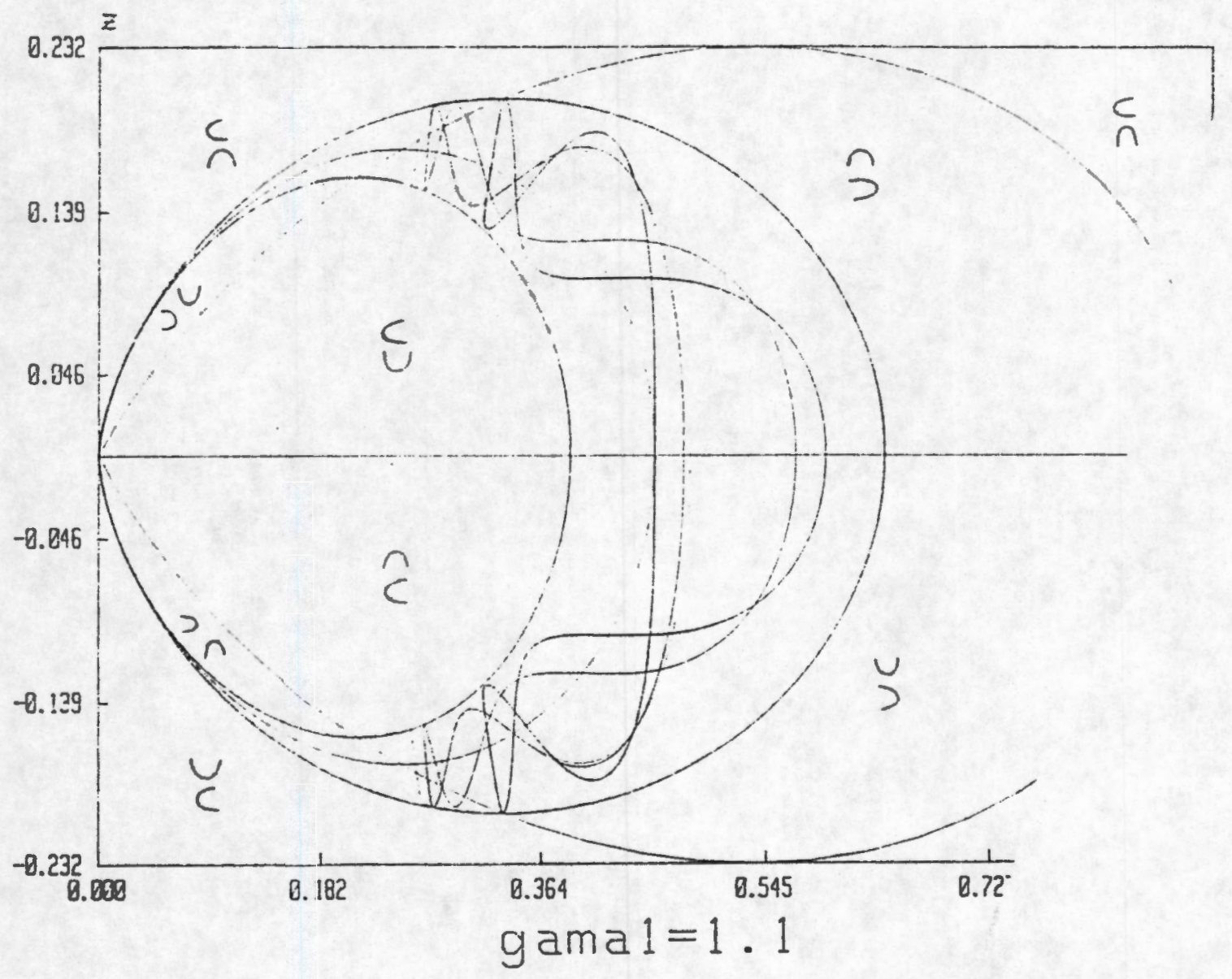

Figura 5.18: Regiones de Graef en el plano meridiano $(\rho, z)$. En cada región se indica el tipo de extremo que puede haber, por ejemplo, $C$ significa que la variable vertical $(z)$ es la variable independiente, y la horizontal $(\rho)$ la dependiente, y que el tipo de extremo es mínimo. Se muestran también cuatro órbitas periódicas simples: $f_{4}$ amarillo, $f_{5}$ morado, $f_{6}$ verde, $f_{7}$ azul. 
del punto $\xi_{1}$ (la órbita que "sale" de la singularidad). También, conforme $m$ aumenta, las trayectorias tienen más oscilaciones alrededor del thalweg en el semiplano inferior. En la figura (5.15) se muestran las órbitas $a_{3,1}, a_{7,2}, a_{8,2}$ y $a_{8,3}$ para $\gamma_{1}=1.1$ 


\section{Capítulo 6}

\section{Órbitas de período 3.}

En este capítulo se hace un seguimiento de la línea $\Gamma_{3}$ al aumentar el parámetro $\gamma_{1}$ para analizar la estructura de las órbitas de período 3 y su clasificación en familias. También se analiza la forma de las órbitas en el plano meridiano. Se encuentra que para una familia dada, permanece esencialmente sin cambio excepto por más ó menos oscilaciones alrededor del thalweg.

\subsection{Clasificación en familias}

Los cálculos numéricos muestran que las familias $f$ y sus bifurcaciones de periodo 3 son esencialmente iguales al aumentar el parámetro $\gamma_{1}$. Esto se debe a que el punto $\xi_{3}$ de $\Gamma_{3}$ orbita alrededor de $\xi_{1}$ (en el sentido de las manecillas del reloj cuando $\gamma_{1}$ aumenta) de tal manera que las intersecciones que se producen en conexión con una familia $f_{2 n}$ son escencialmente las mismas que se producen en conexión con la $f_{2 n+2}$. Además, para $\gamma_{1}>1$ los puntos terminales de las líneas de simetría en la frontera de la región permitida se "desenredan" (desplazándose en el sentido de las manecillas del reloj a lo largo de la frontera $E$ al aumentar $\gamma_{1}$ ) de tal manera que dejan la misma disposición exterior de las líneas al pasar de una familia $f_{2 n}$ a la siguiente $f_{2 n+2}$.

Por otra parte, el análisis de estabilidad de las familias $f_{2 n}{ }^{\prime}$ muestra que son estables en dos segmentos: el que va del máximo de $f_{2 n}$ al punto de intersección con $f_{2 n+1}$, y el segmento final cercano a la frontera de la región prohibida en el espacio $\Sigma$. Cada uno de estos segmentos bombeará órbitas de 
período 3 por el mecanismo descrito en la sección 5.1 (ver figura descriptiva (5.13)). Presentaré aquí las órbitas de periodo 3 conectadas con $f_{0}$ al cambiar $\gamma_{1}$, en el entendido de que esta misma dinámica se presenta para las familias de período 3 conectadas con cualquier familia $f_{2 n}$. Algunas de esas familias ya fueron estudiadas por Markellos et. al. [30] en un pequeño intervalo, y se les llama (en forma genérica) las ramas $j_{3}, j_{3}{ }^{\prime}$ y $k_{3}$ de las familias $j$ y $k$.

En las figuras (6.1) a (6.3) se muestran las líneas $\Gamma_{0}, \Gamma_{1}, \Gamma_{2}$ y $\Gamma_{3}$ para valores crecientes del parámetro. Siguiendo la dinámica de las líneas al aumentar $\gamma_{1}$, asi como los cruces de $\Gamma_{3}$ con $\Gamma_{0}$, se puede obtener una gráfica cualitativa de las órbitas de periodo 3 y su clasificación en familias en e] espacio de parámetros $\Sigma$. En la figura (6.4) se muestra el espacio $\Sigma$ para períodos 1,2 y 3 conectados con $f_{0}$ y $f_{2}$. Como se verá, para cada familia $f_{2 n}$ se obtienen seis ramas diferentes, cuatro de las cuales están reportadas en [30] aunque no en forma global, sino únicamente en la vecindad de $f_{2 n}$ estable.

Para $\gamma_{1}=0.8(C=0.076293)$, sólo una de las dos espirales de $\Gamma_{3}$ se ha intersectado con $\Gamma_{0}$, en dos puntos por los que también pasa $\Gamma_{1}$ (ver figura (5.4)). Estos puntos son las dos órbitas de periodo 1 que definen a $f_{0}$ para ese valor de $\gamma_{1}$. Al aumentar $\gamma_{1}$ se observa la dinámica ya descrita en la sección anterior, por la cual bifurca una pareja (que da dos órbitas diferentes de período 3: una del lado izquierdo de $f_{0}^{\prime}$ (rama a) y otra del lado derecho (rama $b$ ). Esta bifurcación es la estudiada en [30] como bifurcaciones " $j_{3}$ " de la rama " $j$ y que se muestra en el recuadro superior de la figura (6.4).

En la figura (6.1) se muestran las líneas $\Gamma_{0}, \Gamma_{1}, \Gamma_{2}$ y $\Gamma_{3}$ cuando $\gamma_{1}=0.85$ $(C=0.05986)$. El punto $f_{0}{ }^{l}$ se ha desestabilizado dando nacimiento a la familia $f_{1} . \xi_{3}$ se ha movido hacia la izquierda y hacia abajo (aunque aún no se ve en la figura), de manera que la otra espiral de $\Gamma_{3}$ está muy próxima a tocar $\Gamma_{0}$ y generar otra familia de periodo 3 que no bifurca de $f_{0}$ y que denotaré por la rama $c$ en el espacio de parámetros (marcada con una flecha).

En la figura (6.2) se muestran las mismas líneas para $\gamma_{1}=0.9(C=$ $0.04762)$. El punto $\xi_{3}$ continúa su rotación alrededor de $\xi_{1}$ generándose nuevas intersecciones entre las dos espirales alrededor de $\xi_{3}$ y $\Gamma_{0}$. Sin embargo, estas nuevas intersecciones no generan más familias que las ya existentes $b$ y $c$, como quedará claro un poco más adelante. Obsérvese que entre $f_{1}{ }^{l}$ y $f_{0}{ }^{\prime}$, otre espiral de $\Gamma_{3}$ ya se ha intersectado con $\Gamma_{0}$, generándose una nueva familia denotada por $\epsilon$ que, como $c$, tampoco bifurca de $f_{0}{ }^{l}$. El siguiente brazo o vuelta de $\Gamma_{3}$ dará lugar, cuando aumente $\gamma_{1}$, a una nueva familia 


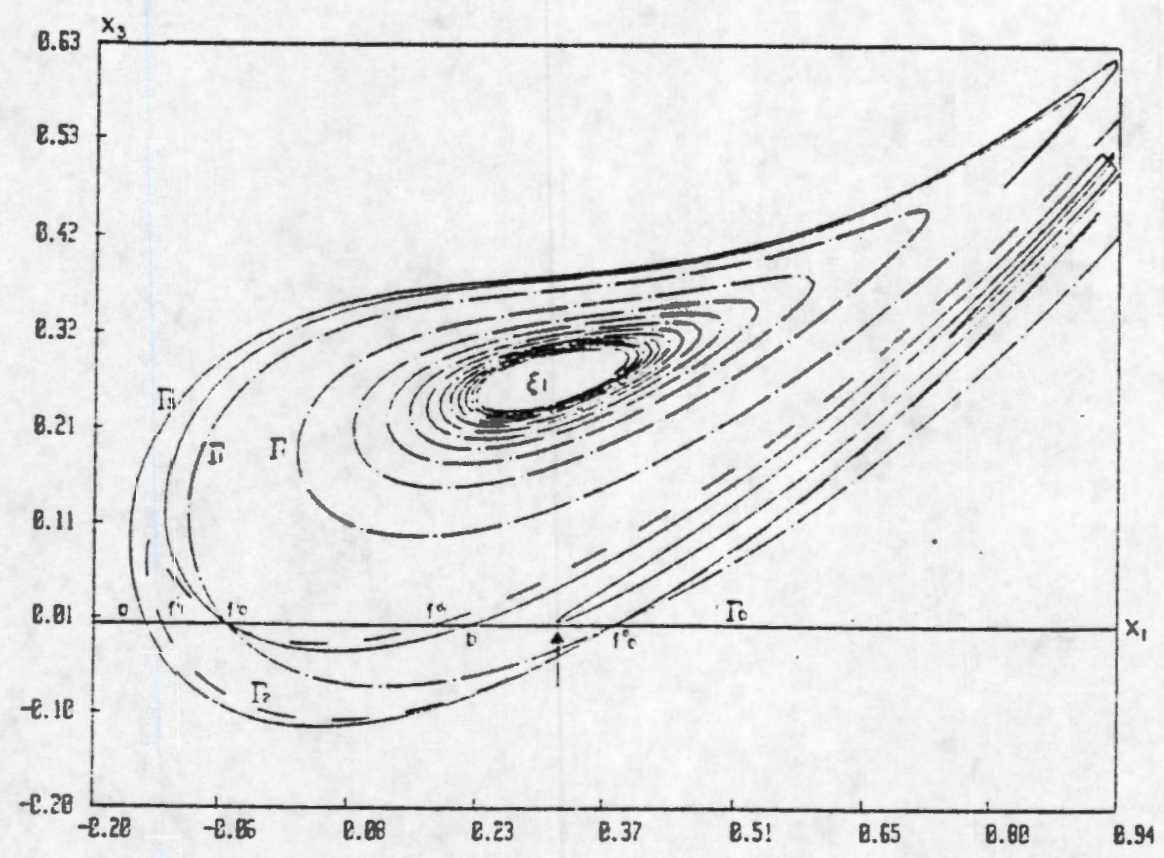

Figura 6.1: Líneas $\Gamma_{0}, \Gamma_{1}, \Gamma_{2}$ y $\Gamma_{3}$ para $\gamma_{1}=0.85(C=0.05986)$. Una nueva familia indicada con una flecha está próxima a nacer (rama $c$ ).

también espiral que denotaré por $g$ pero que aún no aparece para $\gamma_{1}=0.9$.

$\mathrm{El}$ punto $\xi_{3}$ "orbita" alrededor de $\xi_{1}$ al aumentar el parámetro, así que eventualmente cruzará por primera vez a $\Gamma_{0}$. En el espacio de parámetros denotaré por $R_{0}{ }^{3}$ a la primera intersección de $\xi_{3}$ con $\Gamma_{0}$ (ver figura (6.4), es decir, el centro del remolino formado por las ramas $b$ y $c$. Para ese valor del parámetro, infinitas parejas de órbitas habrán nacido entre $f_{1}{ }^{\top}$ y $f_{0}{ }^{r}$, y en adelante comenzarán a desaparecer parejas de órbitas, tanto de la familia $b$ como $c$.

Las parejas de periodo 3 que nacen entre las órbitas $f_{1}{ }^{r}$ y $f_{0}{ }^{r}$ no generan nuevas familias ya que $\Gamma_{3}$ es continua excepto en el punto $\xi_{3}$, con dinámica continua respecto a la variación de $\gamma_{1}[11]$, así que todas las intersecciones que se produzcan entre una sola espiral de $\Gamma_{3}$ con $\Gamma_{0}$, están conectadas continuamente al cambiar $\gamma_{1}$ y formarán una sola familia. Puesto que $\Gamma_{3}$ consta de dos espirales, habrán dos familias que sólo se tocan en el punto $R_{0}{ }^{3}$ en la figura (6.4). Éstas son la rama $b$ que bifurcó de $f_{0}{ }^{l}$ y que termina en $R_{0}{ }^{3}$, y la rama $c$ que no bifurca de $f_{0}{ }^{l}$ pero que también espiralea alrededor de $R_{0}{ }^{3}$ en el espacio de parámetros $\Sigma$. Los cálculos numéricos muestran que $c$ termina ecuatorialmente. 


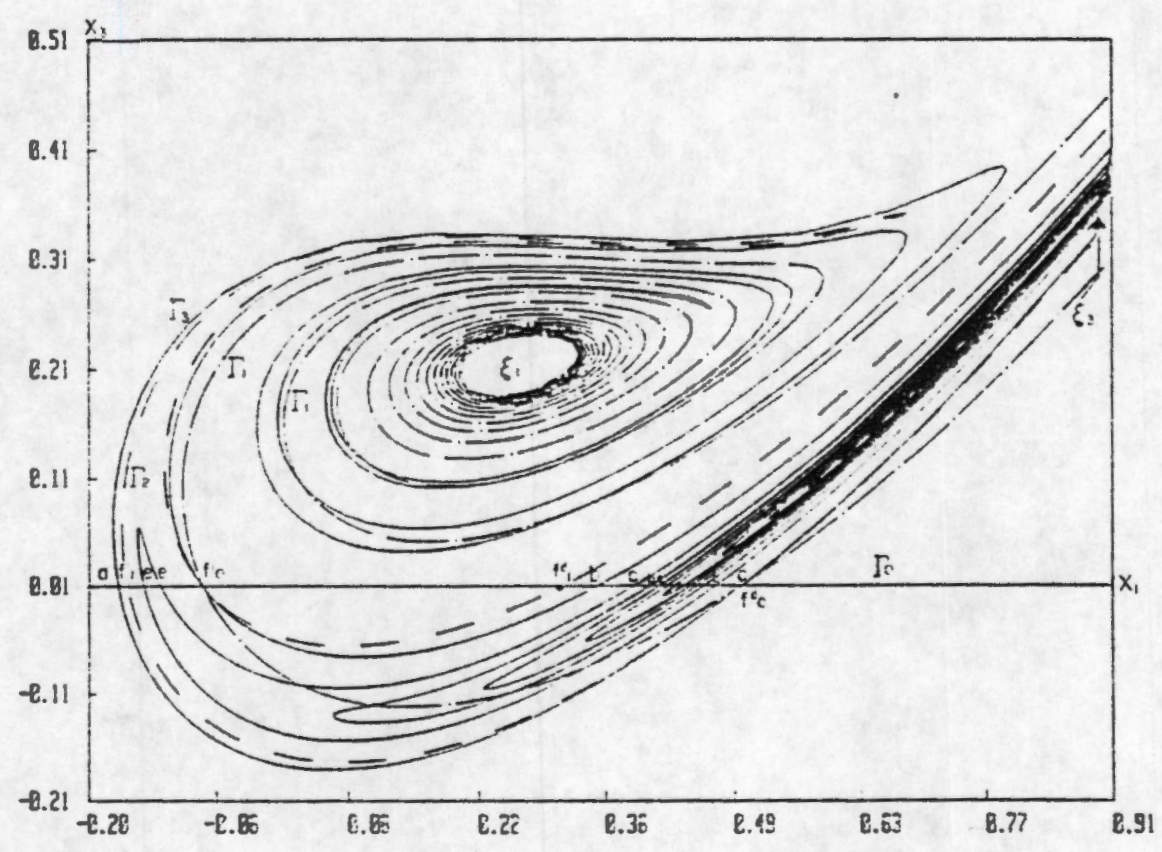

Figura 6.2: Líneas $\Gamma_{0}, \Gamma_{1}, \Gamma_{2}$ y $\Gamma_{3}$ para $\gamma_{1}=0.9(C=0.04762)$. Una nueva familia (rama $e$ de la figura (9) ha nacido entre $f_{1}^{l}$ y $f_{0}{ }^{\prime}$. Las familias $a, b$, $c, e, f_{0}$ y $f_{1}$ se encuentran indicadas. 


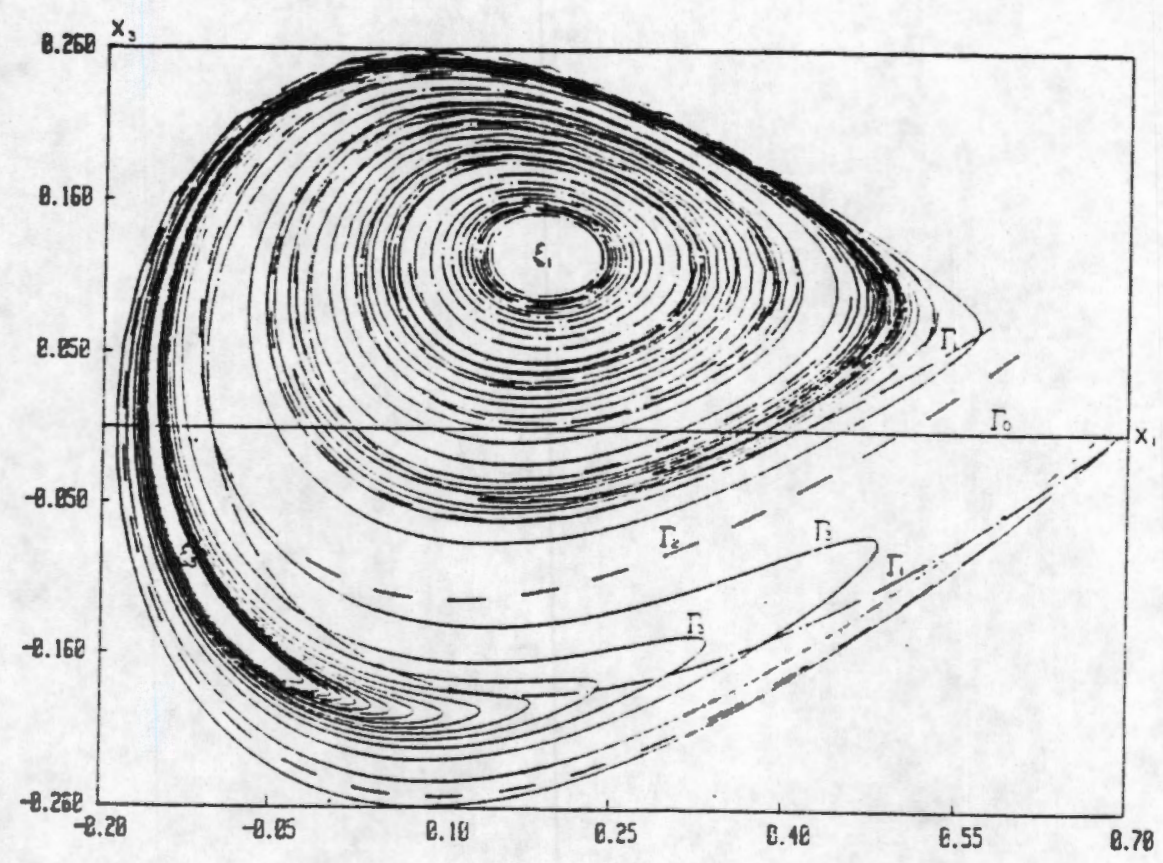

Figura 6.3: Líneas $\Gamma_{0}, \Gamma_{1}, \Gamma_{2}$ y $\Gamma_{3}$ para $\gamma_{1}=1.0(C=0.03125)$.

Obsérvese también que un par de órbitas que nacen juntas pertenecientes a las ramas espirales $b$ o $c$, no desaparecerán juntas: la del lado derecho desaparece con una órbita que nació anteriormente (ie. para un valor de $\gamma_{1}$ menor) del lado izquierdo debido a una intersección de una vuelta más externa del mismo brazo de $\Gamma_{3}$ con $\Gamma_{0}$. Similarmente para las ramas e y $g$, la órbita del lado izquierdo desaparece con una órbita que nace antes del lado derecho. Esta situación ya se ha analizado con respecto a la familia de órbitas asimétricas simples en la sección $\mathbf{5 . 3}$.

El último valor de $\gamma_{1}$ para el que se presenta en forma gráfica a $\Gamma_{3}$ es para $\gamma_{1}=1.0(C=0.03125)$ en la figura (6.3), ya que para valores superiores la gráfica se vuelve muy compleja. Puesto que aparecerán órbitas de período 3 en conexión con $f_{2}$, denotaré por $a_{2 n}, \ldots, g_{2 n}$ a las ramas asociadas a las familias $f_{2 n}$; Este subindice también se utilizará en $R_{2 n}$ ( $n$ entero positivo o cero).

Cuando $\gamma_{1}=1.0$, el punto $\xi_{3}$ ha continuado su movimiento alrededor de $\xi_{1}$ de manera que han desaparecido todas las parejas de las familias espirales $b_{0}$ y $c_{0}$ (entre $f_{1}{ }^{\top}$ y $f_{0}{ }^{\top}$ ) excepto una órbita perteneciente a $c_{0}$ y que está muy próxima a desaparecer ecuatorialmente cuando se desenreden las líneas para $\gamma_{1}>1$. 
Por otro lado, entre $f_{1}{ }^{l}$ y $f_{0}{ }^{\prime}$ se han producido muchisimas intersecciones entre la doble espiral de $\Gamma_{3}$ y $\Gamma_{0}$, pues aparentemente el punto $\xi_{3}$ se encuentra muy cerca de $\Gamma_{0}$. Estas intersecciones pertenecen a las ramas $\epsilon_{0}$ y $g_{0}$ que espiralean alrededor del punto que denoto por $L_{0}{ }^{3}$ en $\Sigma$ y que coresponde a la segunda intersección de $\xi_{3}$ con la línea $\Gamma_{0}$ en su movimiento "orbital" alrededor de $\xi_{1}$ al aumentar $\gamma_{1}$.

La órbita perteneciente a la rama $a_{0}$ (que bifurcó de $f_{0}{ }^{l}$ cerca del máximo en el espacio de parámetros) está aún presente y es la primera intersección entre $\Gamma_{0}$ y $\Gamma_{3}$ de izquierda a derecha en la figura (6.3). Las familias $f_{2}$ y $f_{3}$ ya están presentes por un mecanismo descrito anteriormente (sección 5.2), asi como las ramas $a_{2}$ y $b_{2}$ de $f_{2}$. Además, la doble espiral de $\Gamma_{3}$ ya ha generado nuevas intersecciones con $\Gamma_{0}$ entre los puntos $f_{3}{ }^{r}$ y $f_{2}{ }^{r}$, que son las órbitas $b_{2}$ y $c_{2}$, asi como las órbitas $\epsilon_{2}$ y $g_{2}$ entre $f_{3}{ }^{l}$ y $f_{2}{ }^{l}$.

Por otro lado, una nueva familia $d_{0}$ nació antes (en un valor de $\gamma_{1}$ menor) que la familia $f_{2}, y$ después que la familia $f_{1}$. Para $\gamma_{1}=1.0, d_{0}$ tiene un punto entre $f_{0}{ }^{\prime}$ y $a_{2}$ y otro entre $f_{2}{ }^{r}$ y $f_{1}{ }^{r}$. Los cálculos numéricos muestran que al aumentar el parámetro el lado derecho de la familia $d_{0}$ termina ecuatorialmente, mientras que el lado izquierdo bifurca de $f_{0}{ }^{l}$ en su parte final, i.e. cerca a la frontera de la región permitida en el espacio de parámetros (bifurcaciones de la rama " $k$ " en [30]).

Sin embargo, las familias $d_{m}$ (aquellas que nacen después que la familia $f_{m}$ y antes que la $f_{m+2}$ ( $m$ par $)$ ), no tienen la misma gráfica cualitativa en el espacio de parámetros al pasar de una familia $f_{m}$ a la $f_{m+2}$. Por ejemplo, sólo hay una familia $d_{0}$ para $f_{0}$, mientras que hay dos independientes para $f_{2}$ : 1) La que por la derecha termina ecuatorialmente y por la izquierda bifurca de $f_{0}{ }^{\prime}$ (en su parte final de la gráfica en el espacio de parámetros). Esta rama será denotada por $d_{2}{ }^{0}$. 2) La que está formada por dos secciones que nacen en valores de $\gamma_{1}$ diferentes pero que al aumentar el parámetro $\gamma_{1}$ se ve que se conectan, primero por la izquierda y después por la derecha. Ver figura (6.5). Esta rama será denotada por $d_{2}{ }^{1}$.

A las ramas $d$ del primer tipo (que terminan ecuatorialmente) las denotaré por $d^{0}, y$ a las del segundo tipo que ni terminan ecuatorialmente ni bifurcan de ninguna familia $f$ por $d^{3}, d^{2}$, etc..

Conforme $\gamma_{1}$ aumenta y van naciendo nuevas familias $f_{m}$, las ramas $a_{m}$, $b_{m}, c_{m}, e_{m}$ y $g_{m}$ ( $m$ par) son escencialmente iguales al cambiar $m$, mientras que las ramas $d_{m}$ aparentemente aumentan en número de familias indepen- 


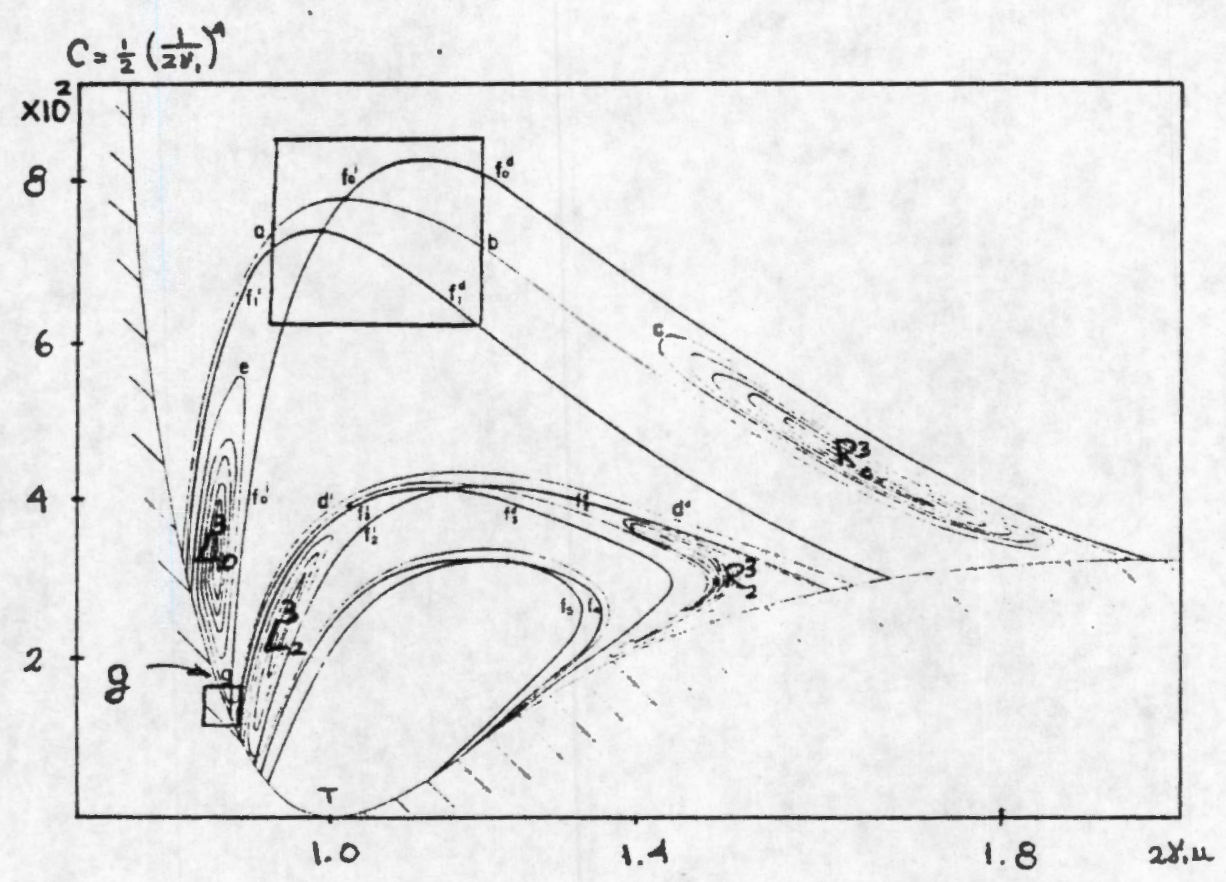

Figura 6.4: Familias $f_{0}, f_{1} \ldots f_{6}$ y las ramas $a, b, c, d$, e y $g$ de periodo 3 asociadas a $f_{0}$ y $f_{2}$ en el espacio de parámetros $C=\frac{1}{2}\left(1 /\left(2 \gamma_{1}\right)\right)^{4}$ contra. $2 \gamma_{3} u=e^{x_{1}}$. Las 6 ramas se han dibujado sólo aproximadamente y se han deformado en la espiral para facilitar el dibujo.

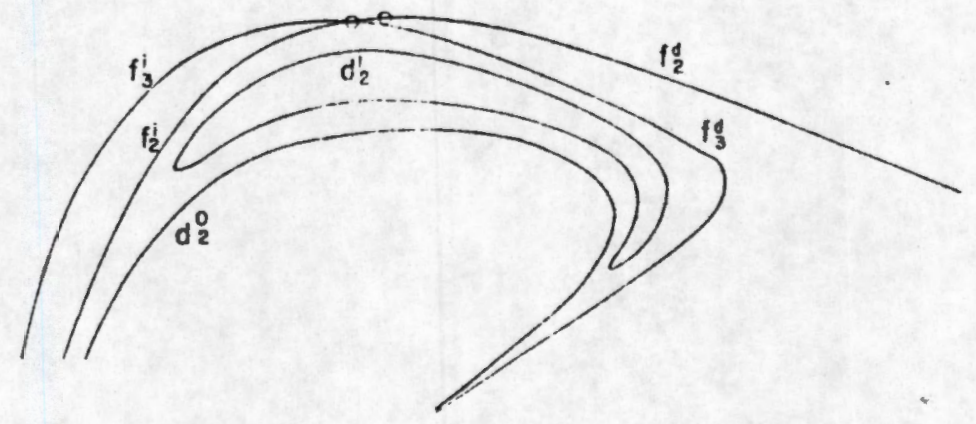

Figura 6.5: Familias $d_{0}^{2}$ y $d_{3}{ }^{2}$. 
dientes $d_{m}{ }^{0}, d_{m}{ }^{1}, d_{m}{ }^{2}$, etc.. Estas requerirán un mayor estudio que el que se presenta aquí .

Las familias a las que pertenecen los puntos de intersección de $\Gamma_{1}, \Gamma_{2}$ y $\Gamma_{3}$ con $\Gamma_{0}$ para $\gamma_{1}=1.0$ (figura (6.3)) son, de menor a mayor valor para la coordenada horizontal $x_{1}$ (ie. de izquierda a derecha):

$$
\begin{aligned}
& a_{0} f_{1}{ }^{\prime} e_{0} g_{0} \varepsilon_{0} g_{0} \ldots \epsilon_{0} g_{0} \epsilon_{0} g_{0} \varepsilon_{0} f_{0}{ }^{l} d_{2}{ }^{0} a_{2} f_{3}{ }^{l} \epsilon_{2} g_{2} g_{2} \epsilon_{2} f_{2}{ }^{\prime} d_{2}{ }^{0} \\
& d_{2}{ }^{1} d_{2}{ }^{1} d_{2}{ }^{0} d_{2}{ }^{0} d_{2}{ }^{1} d_{2}{ }^{1} f_{3}{ }^{\top} b_{2} c_{2} b_{2} c_{2} c_{2} b_{2} c_{2} f_{2}{ }^{\top} d_{0}{ }^{0} f_{1}{ }^{r} c_{0} f_{0}{ }^{r}
\end{aligned}
$$

Se calcularon las líneas $\Gamma_{1}$ a $\Gamma_{3}$ para valores $\gamma_{1}>1$ pero no se presentan aqui debido a su complejidad. Los resultados correspondientes a las familias de periodo 3 en conexión con $f_{0}$ y $f_{2}$ se muestran cualitativamente en la figura (6.4), y cabe remarcar lo siguiente:

1. La familia $f_{2 n}{ }^{l}$ vuelve a ser estable en su segmento final cercano a la frontera de la región prohibida (recuadro inferior de la figura (6.4)). $\mathrm{Si}$ ahora se analiza en forma inversa, es decir cuando $\gamma_{1}$ disminuye, se observa escencialmente la misma dinámica que en la parte estable cercana al máximo de $f_{2 n}{ }^{l}$ : ésta bombea órbitas periódicas de todos los períodos. La pareja perteneciente a las ramas $g_{2 n}$ y $d_{2 n}{ }^{0}$ que bifurca de $f_{0}{ }^{\prime}$ forman una familia.

2. La familia $e$ termina ecuatorialmente. Esto se debe a que el punto final de la espiral correspondiente de $\Gamma_{3}$ (que es un punto sobre la frontera de la región permitida en el plano de Poincaré) rota en el sentido de las manecillas del reloj al aumentar $\gamma_{1}$, hasta que pasa por $\Gamma_{0}$. En ese momento la órbita se vuelve ecuatorial.

3. La rama $a$ termina ecuatorialmente.

4. Las ramas $a$ y $b$ pertenecen a la misma familia.

5. Las ramas $b$ y $c$ podrían considerarse de la misma familia si se considera que se conectan en el punto $R^{3}$. Este punto corresponde a la trayectoria que sale de la singularidad y cruza por segunda vez el plano de Poincaré con $x_{3}=0$, i.e. ortogonalmente al ecuador. Lo mismo pueden considerarse $e$ y $g$ de la misma familia si se considera que se conectan en $L^{3}$. Asi $a, b$ y $c$ formarian una sola familia que bifurca de $f_{0}{ }^{\prime}$ cerca de su máximo, y $d^{0}, \epsilon$ y $g$ otra familia que bifurca de $f_{0}^{\prime}$ cerca de la frontera con la región prohibida. 
6. Las variedades dibujadas en el espacio de parámetros sólo se intersectan en puntos de bifurcación.

7. En [31] sólo se reportan las partes cercanas a $f_{0}^{\prime}$ estables, que se muestran en los recuadros de la figura (6.4) y que sólo incluyen pequeños segmentos de las ramas $a, b$ y $g, d^{0}$. Aquí las he extendido a todo el espacio de parámetros obteniendo la forma espiral de $b$ y $g$, asi como dos nuevas ramas: la $e$ y la $c$, también espirales, y que no se habjan reportado anteriormente. También se ha visto que $a, c, d^{0}$ y $e$ terminan ecuatorialmente, $y$ se han encontrado nuevas familias $d^{1} y d^{2}$ que no bifurcan de $f_{0}$ ni terminan ecuatorialmente.

\subsection{Forma de las trayectorias de periodo 3}

Las órbitas que pertenecen a la misma familia cambian su forma en el espacio de configuración de manera continua. Cuando la rama $a$ bifurca de $f_{0}{ }^{l}$ tiene la forma $\alpha$, y ésta se mantiene semejante a lo largo de toda la rama. La rama $b$ de $f_{0}{ }^{l}$ tiene la forma $b$ cuando bifurca de $f_{0}{ }^{l}$ y la mantiene prácticamente sin cambio a lo largo de la parte externa de la espiral. Las órbitas que se producen por la siguiente intersección de una vuelta más interna de $\Gamma_{3}$ con $\Gamma_{0}$, dá órbitas que tienen una oscilación más alrededor del thalweg en sus extremos que tocan la curva de velocidad cero. Órbitas muy cercanas a $R^{3}$ efectuarán un gran número de oscilaciones alrededor del thalweg hasta tocar la curva de velocidad cero y regresar sobre sí mismas (en el espacio de configuración).

Aunque los extremos de las órbitas que tocan la curva de velocidad cero pueden tener más o menos oscilaciones alrededor del thalweg para una familia. espiral de periodo 3 , la forma básica de la familia no cambia. Así , para las ramas $b$ y $c$ de $f_{0}^{l}$ la forma básica es $b$. De hecho, el punto $R^{3}$ corresponde a una órbita de período 3 con la forma básica $\mathcal{X}$ y que llega a la singularidad.

La forma básica de las ramas $e$ y $g$ de $f_{0}^{\prime}$ es $\varnothing$ y el punto $L^{3}$ corresponde a una órbita con esa forma básica que llega a la singularidad.

Algunos aspectos que requieren más estudio o quedan por resolver son:

1. Analizar la estabilidad de las ramas aquí presentadas. En la región (o regiones) donde se encuentre estable, se tendrán órbitas con periodo 
múltiplo de 3.

2. Calcular las líneas $\Gamma_{4}, \Gamma_{5}$, etc y estudiar su dinámica al cambiar el parámetro. Se espera que las familias de periodo impar tengan espirales. Es posible que haya un comportamiento común que permita entender en forma global la estructura de órbitas con periodo bajo. También es factible que las ramas de las familias de periodo $m$ asociadas a $f_{2 n}$ tengan conexión directa con la forma cualitativa de $f_{0}$.

3. Estudiar con precisión el movimiento de $\xi_{1}, \xi_{3} \ldots, \xi_{2 n+1} \ldots$ al aumentar el parámetro, pues esto dará información sobre la posición de los centros de las familias espirales en el espacio de parámetros $\left(R^{3}\right.$ y $\left.L^{3}\right)$.

4. Estudiar las líneas de simetría en el limite en que $\gamma_{1} \rightarrow \infty(C \rightarrow 0)$ 


\section{Conclusiones}

En sistemas Hamiltonianos con dos grados de libertad y una constante de movimiento para los que se puede definir una sección de Poincaré, puede obtenerse mucha información si el operador de evolución temporal en el plano de Poincaré se puede factorizar en el producto de dos involuciones. Órbitas periódicas simétricas y asimétricas se pueden obtener y analizar por medio de las líneas de simetria, que como hemos visto, contienen una gran información acerca del nacimiento (ó desaparición) y bifurcaciones de estas órbitas.

Además la clasificación de las órbitas periódicas en familias en el contexto de las líneas de simetría es muy natural. Un hecho importante de notar es que las líneas de simetría proporcionan información global del sistema dinámico. 


\section{Agradecimientos}

Deseo expresar mi agradecimiento al Dr. Eduardo Piña por sus valiosos comentarios y discusiones. También quiero agradecer profundamente a Eduardo Neve, Lalito, Ceci y Georgina Lara su continuo apoyo e impulso durante la elaboración de este trabajo.

Finalmente, quiero agradecer a Joaquín Delgado, Ernesto Pérez, Ernesto Lacomba, Arturo Olvera y Jose Luis Córdoba por sus útiles discusiones, y muy especialmente a Javier Jiménez por su ayuda en el aspecto computacional. 


\section{Bibliografía}

[1] Alfvén, H: (1950), Cosmical Electrodynamics, Clarendon Press, Oxford

[2] Birkhoff, G. D.: (1927), Dynamical Systems, AMS. Coll. Pub. 9

[3] Birkhof, G. D.: (1950) Coll. Math. Papers AMS. Vol. I y II

[4] Braun, M.: (1970), Indiana Univ. Math. J. 20 5, 469

[5] Braun, M.: (1970), J. Diff. Equat. 8, 294

[6] Braun, M.: (1970), Ind. Univ. Math. Jour. 20, (5),469

[7] Chavoya, O. and Piña, E.: (1988) Rev. Mex. de Fís. 34, No.1, 32

[8] Chavoya, O. and Piña, E.: (1989), Nuov. Cimen. 103B, 369

[9] Devaney, R. L.: (1976), Trans. Am. Math. Soc. 218, 89

[10] De Vogelaere, R.: (1950), Can.J.Math. 2, 440

[11] De Vogelaere, R.: (1958) in Lefschetz S. Contributions to the Theory of Non-linear Oscillations, Princeton Univ. Press, vol 4, 53

[12] Dragt, A. and Finn, J.: (1976), J. Geophys. Res. 81, 2327

[13] Gardner, C.S.: (1962), On the Conteinment for the Infinite Times of Particles in a Mirror Machine, New York Univ.

[14] Godart, O.: (1938), Ann. Soc. Sci. Brux. 58, $2 T$

[15] Goudas, C., Halioulias, A., Markellos, V. and Macris, G.: (1976) Long Time Predictions in Dynamics Szebehely and Tapley eds., Reidel Pub. Comp., Dordrecht-Holland, 267 
[16] Graef, C. and Kusaka, S.: (1938), J. Math. Phys. 17, 43

[17] Graef, C.: (1944), Boletín de la Soc. Mat. Mex. I 3, I

[18] Green, J. M.: (1979), J. Math. Phys. 20(6), 1183

[19] Jackson, J. D.: (1975) Classical Electrodynamics J. Wiley, N. Y., p. 588

[20] Jiménez-Lara, L. and Pina, E.: aceptado en Celest. Mech.

[21] Jiménez-Lara, L.: aceptado en la Rev. Mex. de Fís.

[22] Jung, C. and Scholz, H. J.: (1988), J. Phys. A.: Math. Gen. 21, 2301

[23] Leage,I. and Mac Kay, R. S.: (1986), Phys. Letters A 118, 274

[24] Lemaitre, G. and Vallarta, M.S.: (1933), Phys. Rev. 43, 87

[25] Lemaitre, G. Ann. de la Soc. Sci. de Brux. (1934) A54, 194

[26] Malmquist, J. (1944), Ark. Math. Astr. och Fysik 30A (5),1

[27] Markellos, V.: (1976), Astrophys. and Spa. Sci.,43, 449

[28] Markellos, V. and Hallioulias A.: (1977), Astrophys. and Spa. Sci. 51, 177

[29] Markellos, V. and Klimopoulos, S.: (1977), Astrophys. and Spa. Sci. 48, 471

[30] Markellos, V, Klimopoulos, S. and Hallioulias, A.: (1978), Celest. Mech. 17, (I) 215

[31] Markellos, V., Klimopuolos, S. and Goudas, C.: (1978), Celest. Mech. 17, (II) 233

[32] Markellos, V.: (1978) Astron. Astrophys. 67, 229

[33] Mavraganis, A. and Goudas C.: (1975), Astrophys. and Spa. Sci. 32, 115

[34] Noguera M.: (1986), Tesis Doctoral, Univ. de Barcelona. 
[35] Northrop, T.G.: (1963), The Adiabatic Motions of Charged Particles, J. Wiley, N.Y.

[36] Piña, E. and Jiménez-Lara, L.: (1987), Physica 26D, 369

[37] Piña, E. and Cantoral, E.: (1989), Phys. Lett. A 135, No.3, 190

[38] Richter, P. H., Scholz, H. J., and Wittek, A., to appear in Nonlinearity.

[39] Störmer, C.: (1907), Arch. Sc. Phys. Nat. 24, 113

[40] Störmer, C.: (1930), Zeits. für Astrophys. 1, 237

[41] Störmer, C.: (1955), The Polar Aurora, Clarendon Press, Oxford.

[42] Vallarta, M.S.: (1938), An Outline of the Theory of the Allowed Cone of Cosmic Radiation, Univ. of Toronto Press. 